\title{
Asymptotic Motion of a Classical Particle in a Random Potential in Two Dimensions: Landau Model ${ }^{\star \star \star}$
}

\author{
Detlef Dürr ${ }^{1 \star}$, Sheldon Goldstein ${ }^{2}$ and Joel L. Lebowitz ${ }^{2 \star \star}$ \\ 1 Fachbereich Mathematik, Ruhr Universität Bochum, Bochum, FRG \\ 2 Department of Mathematics, Rutgers University, New Brunswick, New Jersey 08903, USA
}

\begin{abstract}
We consider the motion of a classical particle in a random isotropic potential arising from uniformly distributed scatterers in two dimensions. We prove that in the weak coupling limit the velocity process of the particle converges in distribution to Brownian motion on a surface of constant speed, i.e. on the circle. The resulting equation for the probability density of the particle is related to the Landau equation in plasmas.
\end{abstract}

\section{Introduction}

We consider the classical motion of a point particle in a random potential $U(\mathbf{x})$ in the van Hove limit: Let $U_{\omega}(\mathbf{x})$ be a realization of the random potential and let $\mathbf{x}(t), \mathbf{v}(t)$ denote position and velocity of the particle given by

$$
\begin{aligned}
\dot{\mathbf{x}}(t) & =\mathbf{v}(t), \quad \mathbf{x}(0)=\mathbf{x}_{0}, \\
\dot{\mathbf{v}}(t) & =-\varepsilon \nabla U_{\omega}(\mathbf{x}(t))=\varepsilon \mathbf{F}_{\omega}(\mathbf{x}(t)), \quad \mathbf{v}(0)=\mathbf{v}_{0} .
\end{aligned}
$$

Clearly $(\mathbf{x}(t), \mathbf{v}(t))_{t \geqq 0}$ is a stochastic process (on the probability space of the random potential). We study the distribution of

as $\varepsilon \rightarrow 0$.

$$
\left(\mathbf{x}^{\varepsilon}(t), \mathbf{v}^{\varepsilon}(t)\right)_{t \geqq 0}, \dot{\mathbf{x}}^{\varepsilon}(t)=\mathbf{v}^{\varepsilon}(t), \mathbf{v}^{\varepsilon}(t)=\mathbf{v}\left(t / \varepsilon^{2}\right)
$$

Kesten and Papanicolaou showed that under some mixing assumption on the (non-conservative) force $\mathbf{F}$ the processes $\left(\mathbf{x}^{\varepsilon}(t), \mathbf{v}^{\varepsilon}(t)\right)_{t \geqq 0}$ converge in distribution to a diffusion process $\left(\mathbf{x}^{0^{\prime}}(t), \mathbf{v}^{0}(t)\right)_{t \geq 0}$ as $\varepsilon \rightarrow 0$ when the space dimension is larger than 2 [1]. (See [2] for a quantum mechanical version.) The two dimensional case was left open.

The dimensionality comes into play since in three or higher dimensions trajectories $\left(\mathbf{x}^{0}(t)\right)$ of the limit process do not intersect themselves, whereas in the

* Heisenberg fellow, also: BiBoS Universität Biclefeld, Bielefeld

$\star \star$ also: Department of Physics

$\star \star \star$ Work supported in part by National Science Foundation Grant No. DMS-85-12505 and AFOSR No. C010 
two dimensional case they typically will. One may convince oneself easily that the presence of self-intersections contribute strongly to the "Non-markovianness" of the motion, thus "making it harder for the limit to become markovian" if at all.

We prove here the convergence also for the two dimensional system. We restrict ourselves for simplicity to the potential case which in addition to having an intrinsic interest of its own can also be considered as a simplified version of a many body system with weak interactions. A particular example of such a system is a plasma where, under suitable conditions, the dominant dynamical effects are due to the Coulomb interactions at "long" distances which can be treated as weak. The system can then be described by a momentum and energy conserving non-linear Fokker-Planck type equation - the Landau equation. A rigorous derivation of that equation is beyond our reach at the present time, but we expect that some of the ideas developed here will also be useful in the many body case with the same type of "Landau scaling," $\varepsilon \rightarrow 0$.

In this limit the potential energy tends to zero and the limit process $\mathbf{v}^{0}$ will satisfy $\left|\mathbf{v}^{0}\right|=\left|\mathbf{v}_{0}\right|$ by conservation of energy. Furthermore we model the random potential $U$ by Poisson distributed scatterers, symmetric finite range potentials $V$. This reduces considerably technicalities in the proof and focuses already on the relevant structure of the motion as arising from many independent scattering events. Instead of scaling the process one may equivalently scale the scatterer field $U$ by

$$
U^{\varepsilon}(\mathbf{x})=\sum_{\mathbf{r}_{i} \in(1)} \varepsilon V\left(\left(\mathbf{x}-\mathbf{r}_{i}\right) / \varepsilon^{2}\right),
$$

$\omega$ representing a configuration of Poisson points with density $\rho^{\varepsilon}=\varepsilon^{-4},\left(\varepsilon^{-2 d}\right.$ in $d$ dimensions).

We consider $\left(\mathbf{x}^{\varepsilon}(t), \mathbf{v}^{\varepsilon}(t)\right)_{t \geqq 0}$, the solutions of

$$
\begin{aligned}
\dot{\mathbf{x}}^{\varepsilon}(t) & =\mathbf{v}^{\varepsilon}(t), \quad \mathbf{x}^{\varepsilon}(0)=\mathbf{x}_{0}, \\
\dot{\mathbf{v}}^{\varepsilon}(t) & =-\nabla U^{\varepsilon}\left(\mathbf{x}^{\varepsilon}(t)\right), \quad \mathbf{v}^{\varepsilon}(0)=\mathbf{v}_{0},
\end{aligned}
$$

and we prove the convergence to a diffusion process $\left(\mathbf{x}^{0}(t), \mathbf{v}^{0}(t)\right)_{t \geqq 0}$, where $\mathbf{v}^{0}$ describes a Wiener process on the circle with radius $v_{0}$.

A brief heuristic argument is as follows: Since $v(t) \sim v_{0}$ the time $\Delta t^{\varepsilon}$ to cross a scattering is roughly $\varepsilon^{2}$. Therefore the change in velocity due to a single scatterer is

$$
\Delta \mathbf{v} \sim \nabla V^{\varepsilon} \cdot \Delta t^{\varepsilon} \sim \varepsilon^{-1} \varepsilon^{2}=\varepsilon .
$$

Assuming that the effect of (the overlapping!) scatterers is approximately additive and noting that the rate at which the particle encounters scatterers is $\sim \rho^{\varepsilon} \varepsilon^{2}=\varepsilon^{-2}$, we obtain that the total variance in time $t$ is

$$
\Delta \mathbf{v}^{2} t \varepsilon^{-2} \sim t .
$$

This Central Limit type argument suggests the Wiener like diffusion on the circle for the velocity. With little extra effort this argument also correctly gives the diffusion constant.

The dimensionality enters if one looks critically at the argument, which involves a weak dependence assumption of the single scattering events. This is drastically 
violated when the particle trajectory intersects itself. One may convince oneself, however, rather quickly that the paths of the limit position process have no tangential self-intersections. Hence presumably also before the limit self-crossings are transversal and happen only finitely often. Thus the total "area of self-crossings" is $\sim \varepsilon^{4}$, and hence only finitely many scatterers are involved in rescatterings. But since the effect of each scatterer is small $(\sim \varepsilon)$ these scatterers should not contribute to the limit.

This simple argument is probabilistic; from analytic arguments given (see [1]) in terms of perturbation expansions, it is not so immediate how to handle non-markovian effects due to (actually occurring) self-crossings. One sees however rather quickly that the effect of scatterers becomes additive.

An earlier announcement of the result presented here describes a proof built on a markovian approximation and convergence of generators [3]. That (very long) proof is different from the one given here. We prove now convergence via relative compactness of the family of measures induced on path space by $\mathbf{v}^{\varepsilon}$ and by the martingale characterization of the limit diffusion [4]. This becomes technically simple through the use of a point process in the description of the motion. The point process describes the places and times at which the particle enters new scatterers. The same technique succeeds in proving the diffusion limit of a heavy particle in an ideal gas in a semi-infinite system with elastic reflection at the boundary in the so-called Brownian motion scaling [5].

In the following section we define the model and give the result. In Sect. II we restate the theorem using stopping times which allow for an easy control of the problematic terms in the convergence. In Sect. III we introduce the point process related to the first entrances of the particle into scatterers, which is then used to express the velocity process. The martingale decomposition of a point process [6], first used in establishing tightness in Sect. IV, plays a central role throughout the proof. In Sect. $\mathrm{V}$ we identify a unique limit process through a martingale characterization. We prove in the appendix that the trajectories of the limit position process do not intersect themselves tangentially. This allows us to remove the stopping times from the convergence result.

\section{Formulation of the Problem}

Let $\left(N, \Omega, F, p^{\varepsilon}\right), \varepsilon>0$, denote a family of Poisson fields in $\mathbb{R}^{2}$ with densities $\rho^{\varepsilon}=\varepsilon^{-4} \rho$, i.e. the expected number of points in a measurable set $A \subset \mathbb{R}^{2}$ is $E^{\varepsilon}(N(A))=\rho|A| \varepsilon^{-4}$. A point $\omega \in \Omega$ represents a realisation of points, i.e. $\omega=\left\{\mathbf{r}_{i}\right\}_{t \in \mathbb{Z}}$, $\mathbb{n}_{i} \in \mathbb{R}^{2}$.

Let $V(x)$ be three times differentiable and supported in $(-1,1)$. Then we set for $\mathbf{x} \in \mathbb{R}^{2}$,

and

$$
V^{\varepsilon}(\mathbf{x})=\varepsilon V\left(|\mathbf{x}| / \varepsilon^{2}\right)
$$

$$
\mathbf{F}^{\varepsilon}(\mathbf{x})=\frac{1}{\varepsilon} \mathbf{F}\left(|\mathbf{x}| / \varepsilon^{2}\right)=-\nabla V^{\varepsilon}(\mathbf{x})
$$

We wish to study the motion of a point particle of mass 1 in the random force 
field obtained by distributing the "scatterers" $V^{\varepsilon}$ according to the Poisson distribution with density $\rho^{\varepsilon}$. The equations of motion for position $\mathbf{x}$ and velocity $\mathbf{v}$ are

$$
\begin{aligned}
& \frac{d \mathbf{x}^{\varepsilon}(t)}{d t}=\mathbf{v}^{\varepsilon}(t), \quad \mathbf{x}^{\varepsilon}(0)=0, \\
& \frac{d \mathbf{v}^{\varepsilon}(t)}{d t}=\sum_{\mathbf{r}_{i} \in \omega} \mathbf{F}^{\varepsilon}\left(\mathbf{x}^{\varepsilon}(t)-\mathbf{r}_{i}\right), \quad \mathbf{v}^{\varepsilon}(0)=\mathbf{v}_{0} .
\end{aligned}
$$

The "overlap lemma" below ensures that the sum in (1.2) is $P^{\varepsilon}$-almost surely finite for all $\varepsilon>0$. Hence (1.1) and (1.2) define uniquely a family of stochastic processes $\left(\mathbf{x}^{\varepsilon}(t), \mathbf{v}^{\varepsilon}(t)\right)_{t \geqq 0}$ on $\left(\Omega, \mathscr{F}, P^{\varepsilon}\right)$.

We wish to study the limit in law of these processes as $\varepsilon \rightarrow 0$. Since $\mathbf{x}^{\varepsilon}(t)$ is obtained from $\mathbf{v}^{\varepsilon}(t)$ by integration we need only consider the convergence in law of $\left(\mathbf{v}^{\varepsilon}(t)\right)_{t \geqq 0}[7]$.

We shall prove

(1.3) Theorem. The family of processes $\left(\mathbf{v}^{\varepsilon}(t)\right)_{t \geqq 0}$ converges as $\varepsilon \rightarrow 0$ in law to a Wiener process $(\mathbf{v}(t))_{t \geqq 0}$ on the circle of radius $v_{0}$ with diffusion constant

$$
D=\frac{\pi \rho}{v_{0}} \int d k k^{2}|\hat{V}(k)|^{2}
$$

and $\hat{V}$ denoting the Fourier transform of $V$.

(1.5) Remark. For $\mathbf{p}=\left(p_{1}, p_{2}\right) \in \mathbb{R}^{2}$, let $\nabla_{p}=\left(\partial / \partial p_{1}, \partial / \partial p_{2}\right), p=|\mathbf{p}|$.

The generator of the limit process $(\mathbf{v}(t))_{t \geqq 0}$ reads on $C_{0}^{\infty}\left(\mathbb{R}^{2}\right)$ :

$$
L=\pi \rho \nabla_{p} \cdot \int d^{2} \mathbf{k} \mathbf{k} \mathbf{k} \delta(\mathbf{k} \cdot \mathbf{p})|\hat{V}(k)|^{2} \cdot \nabla_{p},
$$

where $\mathbf{k} \mathbf{k}$ denotes the tensor product. In polar coordinates $\mathbf{p}=(p, \phi)$,

$$
L=D \partial^{2} / \partial \phi^{2} \text {. }
$$

\section{A Stopping Time Version}

We choose an arbitrary $T>0$ and prove convergence in law of $\left(\mathbf{v}^{\varepsilon}(t)\right)_{t \in[0, T]}=\left(\mathbf{v}^{\varepsilon}(t)\right)$. We shall introduce stopping times which may be removed for $(\mathbf{v}(t))$ and prove the theorem for the stopped process.

First we realize the processes $\left(\mathbf{v}^{\varepsilon}(t)\right)$ and $(\mathbf{v}(t))$ on $C[0, T]^{2}, \mathscr{B}\left(C[0, T]^{2}\right)$ with induced measures $v^{\varepsilon}, v$. The convergence in law of $\left(\mathbf{v}^{\varepsilon}(t)\right)$ to $(\mathbf{v}(t))$ means weak convergence of the measures $v^{\varepsilon}$ to $v$ as $\varepsilon \rightarrow 0$ [7].

For $\mathbf{p} \in C[0, T]^{2}$, let $\mathbf{Q}(t)=\int_{0}^{t} \mathbf{p}(u) d u$ and define for any $a>0$ and angle $\phi$,

$$
\begin{aligned}
\tau_{\phi, a}= & \inf \{t ; \text { there exists } s ; t \geqq s \geqq 0 \quad \text { and } \quad|\mathbf{Q}(s)-\mathbf{Q}(t)| \leqq a \\
& \text { and } \left.\min _{\varsigma \leqq u \leqq t} \mathbf{p}(s) \cdot \mathbf{p}(u) \leqq 0 \text { and } \frac{|\mathbf{p}(t) \cdot \mathbf{p}(s)|}{p(t) p(s)} \geqq \cos \phi\right\}
\end{aligned}
$$


If the set is empty we set $\tau_{\phi, a}=T$.

For $K \in \mathbb{N}$ set

$$
\begin{aligned}
\tau_{K}= & \inf \left\{t \geqq 0 ; \text { there exist } s_{1}<t_{1}, s_{2}<t_{2}, \ldots, s_{K}<t_{K},\right. \\
& \left.t=t_{K}>\cdots>t_{1}, \text { for which } \mathbf{Q}\left(s_{1}\right)=\mathbf{Q}\left(t_{1}\right), \ldots, \mathbf{Q}\left(s_{K}\right)=\mathbf{Q}\left(t_{K}\right)\right\} .
\end{aligned}
$$

If the set is empty we set $\tau_{K}=T$.

Finally let

$$
\tau_{v}=\inf \left\{t \geqq 0 ;\left|p(t)-v_{0}\right| \geqq v_{0} / 2\right\} .
$$

Clearly $v\left(\tau_{v} \leqq T\right)=0$ and the self-crossing lemma in the appendix gives that

$$
\lim _{\substack{\phi \rightarrow 0 \\ a \rightarrow 0}} v\left(\tau_{\phi, a} \leqq T\right)=0, \quad \lim _{K \rightarrow \infty} v\left(\tau_{K} \leqq T\right)=0 .
$$

Let

$$
\tau=\tau_{v} \wedge \tau_{\phi, a} \wedge \tau_{K}
$$

and define on $C[0, T]^{2}$ a map $\Pi$ by $\Pi \mathbf{p}(t)=\mathbf{p}(t \wedge \tau)$. Then we show that $\tilde{v}^{\varepsilon}=v^{\varepsilon} \cdot \Pi^{-1}$ converges weakly $(\rightarrow)$ to $\tilde{v}=v \cdot \Pi^{-1}$, i.e.,

(2.6) Lemma. $\tilde{v}^{\varepsilon} \rightarrow \tilde{v}$ as $\varepsilon \rightarrow 0$.

The assertion of Theorem (1.3) follows from (2.6) and (2.4) by standard arguments. (See also [1] for the "removal" of similar stopping times.)

There is a natural representation of the stopping times on $\left(\Omega, \mathscr{F}, P^{\varepsilon}\right)$ using the process $\left(\mathbf{v}^{\varepsilon}(t)\right)$. The stopping times become $\varepsilon$-depedent: $\tau_{\phi, a}^{\varepsilon}, \tau_{K}^{\varepsilon}, \tau_{v}^{\varepsilon}$ and $\tau^{\varepsilon}$, and we define the stopped process $\left(\mathbf{v}^{\varepsilon}\left(t \wedge \tau^{\varepsilon}\right)\right) \equiv\left(\mathbf{v}_{t}^{\varepsilon}\right)$. Note that $\tilde{v}^{\varepsilon}$ is the measure induced by $\left(\mathbf{v}_{t}^{\varepsilon}\right)$. We define $\left(\mathbf{x}_{t}^{\varepsilon}\right)$ by $\mathbf{x}_{t}^{\varepsilon}=\int^{t \wedge \tau^{\varepsilon}} \mathbf{v}_{s}^{\varepsilon} d s$.

We may describe the features of the stopped process as follows.

(i) When the particle trajectory $\mathbf{x}_{t}^{\varepsilon}$ bends around and comes as close as $a$ to its past trajectory, then it will cross itself transversally with an angle larger than $\phi$.

(ii) The trajectory does not cross itself more than $K$ times.

(iii) The speed of the particle is between $v_{0} / 2$ and $3 v_{0} / 2$.

We shall prove (2.6) by first establishing tightness of the family $\left(\tilde{v}^{\varepsilon}\right)_{\varepsilon}$. The existence and determination of the limit will then be obtained from the Stroock-Varadhan martingale characterization of diffusion processes. In both steps we shall exploit the fact that the particle motion is determined by the "almost Poisson" point process related to the times and places at which the particle enters new scatterers. We introduce this point process next.

\section{The Point Process Description}

The reader should observe that for the following discussion the stopped version of the process is not needed except for convenience to later references.

Let

$$
T_{t}^{\varepsilon}=\left\{\mathbf{x} \in \mathbb{R}^{2} ;\left|\mathbf{x}-\mathbf{x}_{u}^{\varepsilon}\right| \leqq \varepsilon^{2}, u \leqq t\right\} .
$$

$T_{t}^{\varepsilon}$ contains all the centers of the scatterers which affected the particle motion until time $t$. 
For $\omega \in \Omega$ let $\hat{\omega}=\omega-\omega_{0}$, where

$$
\omega_{t}=\omega \cap T_{t}^{\varepsilon}
$$

and for $\mathbf{r}_{i} \in \hat{\omega}$ let $t_{i}$ be the first hitting time

$$
t_{i}=\inf \left\{t>0 ;\left|\mathbf{x}_{t}^{\varepsilon}-\mathbf{r}_{i}\right| \leqq \varepsilon^{2}\right\} .
$$

We associate with $\left(\Omega, \mathscr{F}, P^{\varepsilon}\right)$ a point process $N^{\varepsilon}$ on $\mathbb{R}^{2} \times \mathbb{R}_{+}$with realizations $N_{\omega}^{\varepsilon}(d \mathbf{r}, d t)$ such that for $f \in C_{0}\left(\mathbb{R}^{2} \times \mathbb{R}\right)$,

$$
N_{\omega}^{\varepsilon}(f)=\int_{\mathbb{R}^{2}} \int_{\mathbb{R}^{+}} N_{\omega}^{\varepsilon}(d \mathbf{r}, d t) f(\mathbf{r}, t)=\sum_{\mathbf{r}_{\mathbf{r}} \in \hat{\omega}} f\left(\mathbf{r}_{i}, t_{i}\right) .
$$

For every measurable $A \subset \mathbb{R}^{2}$, the collection

$$
N_{\omega}^{\varepsilon}(A, t)=\int_{0}^{t} N_{\omega}^{\varepsilon}(A, d u)=N_{\omega}^{\varepsilon}(A,(0, t))=\sum_{\mathbf{r}_{i} \in \omega_{t}-\omega_{0}} X_{A}\left(\mathbf{r}_{i}\right)
$$

$\omega \in \Omega$, defines an increasing right continuous jump process in $t$. Note that the last statement requires proof, which consisting of standard "existence of dynamics" type arguments, is omitted.

We use the notation $N^{\varepsilon}\left(A, t_{-}\right)=N^{\varepsilon}(A,(0, t))$ for the left continuous version of the process.

For every $\omega, N_{\omega}^{\varepsilon}(d \mathbf{r}, d t)$ describes the positions of the scatterer as well as the times of first entry. Note that the process $N^{\varepsilon}$ is locally Poisson with rates depending on the past trajectory of the particle.

If we look at the world as seen from the particle, the above point process is conveniently described by a point process on $S \times \mathbb{R}_{+}, S$ being the unit circle, where we collect now the points $t_{i}$ and $\sigma_{i} \in S$ at which the particle enters the scatterer located at $\mathbf{r}_{i}$. The realizations are now measures on $S \times \mathbb{R}_{+}$, denoted by $N^{\varepsilon}(d \boldsymbol{\sigma}, d t)$. Clearly both point processes are isomorphic to each other.

We introduced the point process because it comes with a decomposition which goes to the heart of the problem:

Let $\left(\mathscr{F}_{t}\right)_{t \geq 0}$ denote the increasing family of $\sigma$-algebras generated by $N^{\varepsilon}(A, s)$, $s \leqq t, A \in \mathscr{B}\left(\mathbb{R}^{2}\right)$ and “ $\omega_{0}$ ". Then for every $\mathscr{F}_{t}$-adapted left continuous (in $t$ ) function $f$ on $S \times \mathbb{R}_{+}$we have that [6]

$$
\begin{aligned}
\int_{S}^{t} \int_{0}^{t} N^{\varepsilon}(d \boldsymbol{\sigma}, d u) f(\boldsymbol{\sigma}, u)= & \int_{S}^{t} \int_{0}^{t} M^{\varepsilon}(d \boldsymbol{\sigma}, d u) f(\boldsymbol{\sigma}, u) \\
& +\int_{S}^{t} \int_{0}^{t} \rho^{\varepsilon}(d \boldsymbol{\sigma}, d u) f(\boldsymbol{\sigma}, u) .
\end{aligned}
$$

where

$$
\int_{S}^{t} \int_{0}^{t} M(d \boldsymbol{\sigma}, d u) f(\boldsymbol{\sigma} \cdot u)=M_{t}(f)
$$

is an $\mathscr{F}_{t}$-martingale and

$$
\int_{S}^{t} \int_{0}^{t} \rho^{\varepsilon}(d \boldsymbol{\sigma}, d u) f(\boldsymbol{\sigma}, u)=\rho_{t}^{\varepsilon}(f)
$$

is an $\mathscr{F}_{t}$ measurable left continuous process. 
Note that $\rho^{\varepsilon}(d \boldsymbol{\sigma}, d u)$ (the compensator of $\left.N^{\varepsilon}[8]\right)$ is the rate at which the particle enters new scatterers. Although it is intuitively quite clear what the rate looks like, one should note that one's intuition uses (correctly so) the strong Markov property [9] of Poisson fields (see also [10]).

We shall now express $\mathbf{v}_{t}$ using the above point process. From (1.2), by integration,

$$
\mathbf{v}_{t}^{\varepsilon}-\mathbf{v}_{0}=\sum_{\mathbf{r}_{i} \in \omega} \int_{0}^{t \wedge \tau^{\varepsilon}} \mathbf{F}^{\varepsilon}\left(\mathbf{x}_{s}^{\varepsilon}-\mathbf{r}_{i}\right) d s
$$

which by (3.2) and (3.3) is the same as

with

$$
\mathbf{v}_{t}^{\varepsilon}-\mathbf{v}_{0}=\sum_{\mathbf{r}_{i} \in \omega_{t}-\omega_{0}} \int_{t_{i} \wedge \tau^{\varepsilon}}^{t \wedge \tau^{\varepsilon}} \mathbf{F}^{\varepsilon}\left(\mathbf{x}_{s}^{\varepsilon}-\mathbf{r}_{i}\right) d s+\Delta \mathbf{v}_{0}^{\varepsilon}(t)
$$

$$
\Delta \mathbf{v}_{0}^{\varepsilon}(t)=\sum_{\mathbf{r}_{i} \in \omega_{0}} \int_{0}^{t \wedge \tau^{\varepsilon}} \mathbf{F}^{\varepsilon}\left(\mathbf{x}_{s}^{\varepsilon}-\mathbf{r}_{i}\right) d s
$$

By (3.4) we may write this as

$$
\mathbf{v}_{t}^{\varepsilon}-\mathbf{v}_{0}=\int_{R^{2}}^{t \wedge} \int_{0}^{\tau^{\varepsilon}} N^{\varepsilon}(d \mathbf{r}, d u) \int_{u}^{t \wedge \tau^{\varepsilon}} \mathbf{F}^{\varepsilon}\left(\mathbf{x}_{s}^{\varepsilon}-\mathbf{r}\right) d s+\Delta \mathbf{v}_{0}^{\varepsilon}(t) .
$$

Let for $\boldsymbol{\sigma} \in S$,

$$
\mathbf{x}_{u, \sigma}^{\varepsilon}(t)=\mathbf{x}_{t}^{\varepsilon}-\mathbf{x}_{u}^{\varepsilon}+\boldsymbol{\sigma} \varepsilon^{2} .
$$

Then (3.8) may also be written as

$$
\mathbf{v}_{t}^{\varepsilon}-\mathbf{v}_{0}=\int_{S}^{t \wedge} \int_{0}^{t \wedge} N^{\varepsilon}(d \boldsymbol{\sigma}, d u) \int_{u}^{t \wedge \tau^{\varepsilon}} \mathbf{F}^{\varepsilon}\left(\mathbf{x}_{u, \sigma}^{\varepsilon}(s)\right) d s+\Delta \mathbf{v}_{0}^{\varepsilon}(t) .
$$

\section{Tightness}

We begin with two estimates.

(4.1) Overlap Lemma. For $n \in \mathbb{N}$, let

$$
S_{n}^{\varepsilon}(t)=\text { disc of radius } n \varepsilon^{2} \text { around } \mathbf{x}_{t}^{\varepsilon},
$$

and let

$$
N_{n}^{\varepsilon}(t)=\left|\omega \cap S_{n}^{\varepsilon}(t)\right| .
$$

Then for any $\lambda>0, T>0$ and $\varepsilon>0$

$$
P^{\varepsilon}\left(\left\{\sup _{t \leqq T} N_{n}^{\varepsilon}(t) \geqq \lambda\right\}\right) \leqq\left[\frac{1+4 \lambda \varepsilon B T}{n \varepsilon^{2}}+1\right]^{2} e^{16 n^{2}(e-1) \rho} e^{-\lambda},
$$

where

$$
B=\sup |V(x)| .
$$

Proof. By conservation of energy (we set $v_{0}=1$ )

$$
\left(v_{t}^{\varepsilon}\right)^{2} \leqq 1+4 \varepsilon \sup _{s \leqq t} N_{1}^{\varepsilon}(s) B .
$$


Thus for

$$
\begin{gathered}
\tau_{\lambda}=\inf \left\{t \geqq 0 ; N_{n}^{\varepsilon}(t) \geqq \lambda\right\}, \\
\left\{\tau_{\lambda}>T\right\} \subset\left\{\sup _{t \leqq T} v_{t}^{\varepsilon} \leqq 1+4 \lambda \varepsilon B\right\} \subset\left\{\sup _{t \leqq T} x_{t}^{\varepsilon} \leqq(1+4 \lambda \varepsilon B) T\right\} .
\end{gathered}
$$

Let for $\mathbf{x} \in \mathbb{R}^{2}$,

and let

$$
S_{n}^{\varepsilon}(\mathbf{x})=\text { disc of radius } n \varepsilon^{2} \text { around } \mathbf{x} \text {. }
$$

$$
N_{n}^{\varepsilon}(\mathbf{x})=\left|\omega \cap S_{n}^{\varepsilon}(\mathbf{x})\right| .
$$

Then for the square $\Gamma=[-(1+4 \lambda \varepsilon B T), 1+4 \lambda \varepsilon B T]^{2}$, we have from the above that

$$
\left\{\tau_{\lambda} \leqq T\right\} \subset\left\{\sup _{\mathbf{x} \in \Gamma} N_{n}^{\varepsilon}(\mathbf{x}) \geqq \lambda\right\} \text {. }
$$

We cover $\Gamma$ by a lattice of squares $\gamma_{i}^{\varepsilon}$ of sides $2 n \varepsilon^{2}, i=1, \ldots,\left[(1+4 \lambda \varepsilon B T) / n \varepsilon^{2}+1\right]^{2}$, so that

Since

$$
\left\{\sup _{\mathbf{x} \in \Gamma} N_{n}^{\varepsilon}(\mathbf{x}) \geqq \lambda\right\} \subset\left\{\sup _{l} \sup _{\mathbf{x} \in \vartheta_{i}^{\varepsilon}} N_{n}^{\varepsilon}(\mathbf{x}) \geqq \lambda\right\}
$$

$$
\sup _{\mathbf{x} \in \gamma_{i}^{\varepsilon}} N_{n}^{\varepsilon}(\mathbf{x}) \leqq N\left(\tilde{\gamma}_{i}^{\varepsilon}\right)
$$

where $\tilde{\gamma}_{i}^{\varepsilon}$ is a square of side $4 n \varepsilon^{2}$ symmetrically covering $\gamma_{i}^{\varepsilon}$, we obtain by translation invariance of the Poisson field that

$$
P^{\varepsilon}\left(\left\{\sup _{t \leqq T} N_{n}^{\varepsilon}(t) \geqq \lambda\right\}\right)=\left[\frac{1+4 \lambda \varepsilon B T}{n \varepsilon^{2}}+1\right]^{2} P^{\varepsilon}\left(N\left(\tilde{\gamma}_{i}^{\varepsilon}\right) \geqq \lambda\right) .
$$

Now (4.2) follows using Markov's inequality for the exponential function.

The second estimate is

(4.3) Crossing Time Lemma. Let $t_{n}^{\varepsilon}(s)$ and $\tilde{t}_{n}^{\varepsilon}(s)$ be the first entrance and exit time of the particle of the set $S_{n}^{\varepsilon}(s)$. Then on

$$
\left\{\sup _{s \leqq T} N_{n+1}^{\varepsilon}(s) \leqq \lambda\right\} \cap\left\{\sup _{s \leqq T} v^{\varepsilon}(s) \geqq 1 / 2\right\},
$$

we have for $\varepsilon<1 / 16 n \hat{\lambda} B^{\prime}$ that

and

$$
\tilde{t}_{n}^{\varepsilon}(s)-t_{n}^{\varepsilon}(s) \leqq 4 n \varepsilon^{2}
$$

$$
\sup _{s \leqq T} \sup _{t_{n}^{\varepsilon}(s) \leqq t \leqq \hat{t}_{n}^{\varepsilon}(s)}\left|\mathbf{v}^{\varepsilon}(t)-\mathbf{v}^{\varepsilon}\left(t_{n}^{\varepsilon}(s)\right)\right| \leqq 4 n \hat{\lambda} \varepsilon B^{\prime}
$$

where

$$
B^{\prime}=\sup |\mathbf{F}(\mathbf{x})|
$$

Proof. By (3.7) for $t \leqq \tilde{t}_{n}^{\varepsilon}(s)$

$$
\left|\mathbf{v}^{\varepsilon}(t)-\mathbf{v}^{\varepsilon}\left(t_{n}^{\varepsilon}(s)\right)\right| \leqq \sum_{\mathbf{r}_{i} \in \omega \cap S_{n+1}^{\varepsilon}(s)}\left|\int_{t_{n}^{\varepsilon}(s)}^{t} \mathbf{F}^{\varepsilon}\left(\mathbf{x}^{\varepsilon}\left(t^{\prime}\right)-\mathbf{r}_{i}\right) d t^{\prime}\right| .
$$

Consider now a particle crossing $S_{n}^{\varepsilon}(s)$ with constant velocity $\mathbf{v}_{1}^{\varepsilon}=\frac{1}{2} \mathbf{v}^{\varepsilon}\left(t_{n}^{\varepsilon}(s)\right)$, entering 
at $t_{n}^{\varepsilon}(s)$ at $\mathbf{x}^{\varepsilon}\left(t_{n}^{\varepsilon}(s)\right)$. Then for the exit time $\hat{t}_{n}^{\varepsilon}(s)$ of this particle

$$
\hat{t}_{n}^{\varepsilon}(s)-t_{n}^{\varepsilon}(s) \leqq \frac{2 n \varepsilon^{2}}{v_{1}^{\varepsilon}} \leqq 4 n \varepsilon^{2},
$$

and for $t<\hat{t}_{n}^{\varepsilon}(s)$ we obtain from (4.6) that

$$
\left|\mathbf{v}^{\varepsilon}(t)-\mathbf{v}_{1}^{\varepsilon}\right| \leqq N_{n+1}^{\varepsilon}(s) \frac{1}{\varepsilon} B^{\prime} 4 n \varepsilon^{2} .
$$

Hence on $\left\{\sup _{s \leqq T} N_{n+1}^{\varepsilon}(s) \leqq \lambda\right\}$ we have that

$$
\mathbf{v}^{\varepsilon}(t) \cdot \frac{\mathbf{v}_{1}^{\varepsilon}}{v_{1}^{\varepsilon}} \geqq v_{1}^{\varepsilon}-N_{n+1}^{\varepsilon}(s) 4 n \varepsilon B^{\prime}>\frac{1}{2} v_{1}^{\varepsilon}
$$

for $\varepsilon$ as stated. Therefore $\hat{t}_{n}^{\varepsilon}(s) \geqq \tilde{t}_{n}^{\varepsilon}(s)$ and (4.4) and (4.5) follow.

We are now prepared to prove

(4.9) Proposition. The family $\left(\tilde{v}^{\varepsilon}\right)_{\varepsilon \geqq 0}$ is tight in $C[0, T]^{2}$.

Recall that (4.9) follows when we show the following: For all $\gamma, \eta>0$ there exists a $\delta$ such that for $\varepsilon$ small enough

$$
\mathrm{P}^{\varepsilon}\left(\left\{\sup _{\substack{s, t \leqq T \\|t-s|<\delta}}\left|\mathbf{v}_{t}^{\varepsilon}-\mathbf{v}_{s}^{\varepsilon}\right|>\gamma\right\}\right)<\eta .
$$

We shall approximate $\mathbf{v}_{t}^{\varepsilon}$ by a process consisting of those terms which are relevant for the limit. The irrelevant terms vanish in probability as $\varepsilon \rightarrow 0$. Therefore (4.10) will in fact be established for the relevant part (see (4.24) below).

Let $S^{\varepsilon}$ denote the circle of radius $\varepsilon^{2}$. We define for $\sigma \in S$

$$
t_{u, \sigma}^{\varepsilon}=\inf \left\{t>u ; \mathbf{x}_{u, \sigma}^{\varepsilon}(t) \notin S^{\varepsilon}\left(\mathbf{x}_{u}^{\varepsilon}-\boldsymbol{\sigma} \varepsilon^{2}\right)\right\} \Lambda \tau^{\varepsilon}
$$

By (3.10)

$$
\begin{aligned}
\mathbf{v}_{t}^{\varepsilon}-\mathbf{v}_{s}^{\varepsilon}= & \int_{S} \int_{s \wedge \tau^{\varepsilon}}^{t \wedge \tau^{\varepsilon}} N^{\varepsilon}(d \boldsymbol{\sigma}, d u) \int_{u}^{t_{u, \sigma}^{\varepsilon}} \mathbf{F}^{\varepsilon}\left(\mathbf{x}_{u, \sigma}^{\varepsilon}\left(t^{\prime}\right)\right) d t^{\prime} \\
& +\int_{S}^{t \wedge \tau_{s}^{\varepsilon}} \int_{\wedge \tau^{\varepsilon}}^{t} N^{\varepsilon}(d \boldsymbol{\sigma}, d u) \int_{t_{u, \sigma}^{\varepsilon}}^{t \wedge \tau^{\varepsilon}} \mathbf{F}^{\varepsilon}\left(\mathbf{x}_{u, \sigma}^{\varepsilon}\left(t^{\prime}\right)\right) d t^{\prime} \\
& +\int_{S}^{s \wedge} \int_{0}^{s \tau^{\varepsilon}} N^{\varepsilon}(d \boldsymbol{\sigma}, d u) \int_{s \wedge \tau^{\varepsilon}}^{t \wedge \tau^{\varepsilon}} \mathbf{F}^{\varepsilon}\left(\mathbf{x}_{u, \sigma}^{\varepsilon}\left(t^{\prime}\right)\right) d t^{\prime} \\
& +\Delta \mathbf{v}_{0}^{\varepsilon}(t)-\Delta \mathbf{v}_{0}^{\varepsilon}(s) .
\end{aligned}
$$

For $\varepsilon \ll a$ (cf. (2.1)), the last three terms concern scatterers which the particle encounters again at occasions of selfcrossings of the particle trajectory and scatterers which at the time $s \wedge \tau^{\varepsilon}$ overlap $\mathbf{x}_{s \wedge \tau^{\varepsilon}}^{\varepsilon}$ or which overlap the particle initially. Recall that until $\tau^{\varepsilon}$ only $K$ selfcrossings are possible and that they are transversal with an angle at least as big as $\phi$. Therefore we can find a number $n(\phi, a)$, not depending on $\varepsilon$, such that by virtue of the crossing time lemma (4.3) on

$$
\left\{\sup _{s \leqq T} N_{n(\phi, a)}^{\varepsilon}(s) \leqq \lambda\right\}
$$

$\sup \mid$ three rightmost terms in $(4.12) \mid \leqq 4 K B^{\prime} n(\phi, a) \lambda \varepsilon$. 
By the overlap lemma (4.1) we may choose $\lambda=\varepsilon^{-\alpha}, \alpha<1$, and obtain that these terms vanish in probability as $\varepsilon \rightarrow 0$.

We consider now the first term on the right of (4.10). We wish to exploit here the point process decomposition (3.6). But note that the point measure integrates here an anticipative function so that (3.6a) and (3.6b) do not hold. Our aim is therefore an approximation of the anticipative function by a non-anticipative one.

We first expand the force $\mathbf{F}^{\varepsilon}$ around

for

$$
\hat{\mathbf{x}}_{u, \sigma}^{\varepsilon}(t)=\mathbf{v}_{u}^{\varepsilon}(t-u)+\boldsymbol{\sigma} \varepsilon^{2}
$$

$$
u \leqq t \leqq \hat{t}_{u, \sigma}^{\varepsilon}=\inf \left\{t>u ; \mathbf{x}_{u, \sigma}^{\varepsilon}(t) \in S^{\varepsilon}\left(\mathbf{x}_{u}^{\varepsilon}-\boldsymbol{\sigma} \varepsilon^{2}\right)\right\} \Lambda \tau^{\varepsilon} .
$$

(Note that (4.13) describes a straight line.)

This yields

$$
\begin{aligned}
\int_{u}^{t_{u, \sigma}^{\varepsilon}} \mathbf{F}^{\varepsilon}\left(\mathbf{x}_{u, \sigma}^{\varepsilon}\left(t^{\prime}\right)\right) d t^{\prime}= & \int_{u}^{\hat{t}_{u, \sigma}^{\varepsilon}} \mathbf{F}^{\varepsilon}\left(\hat{\mathbf{x}}_{u, \sigma}^{\varepsilon}\left(t^{\prime}\right)\right) d t^{\prime} \\
& +\int_{u}^{\hat{t}_{u, \sigma}^{\varepsilon}}\left(\mathbf{x}_{u, \sigma}^{\varepsilon}\left(t^{\prime}\right)-\hat{\mathbf{x}}_{u, \sigma}^{\varepsilon}\left(t^{\prime}\right)\right) \cdot \nabla \mathbf{F}^{\varepsilon}\left(\hat{\mathbf{x}}_{u, \sigma}^{\varepsilon}\left(t^{\prime}\right)\right) d t^{\prime} \\
& +\int_{\hat{t}_{u, \sigma}^{\varepsilon}}^{t_{u, \sigma}^{\varepsilon}} \mathbf{F}^{\varepsilon}\left(\mathbf{x}_{u, \sigma}^{\varepsilon}\left(t^{\prime}\right)\right) d t^{\prime} \\
& +\int_{u}^{\hat{t}_{u, \sigma}^{\varepsilon}} \frac{1}{2}\left(\left(\mathbf{x}_{u, \sigma}^{\varepsilon}\left(t^{\prime}\right)-\hat{\mathbf{x}}_{u, \sigma}^{\varepsilon}\left(t^{\prime}\right)\right) \cdot \nabla\right)^{2} \mathbf{F}^{\varepsilon}\left(\mathbf{y}^{\varepsilon}\left(u, \boldsymbol{\sigma}, t^{\prime}\right)\right),
\end{aligned}
$$

with $\mathbf{y}^{\varepsilon}\left(u, \boldsymbol{\sigma}, t^{\prime}\right)$ denoting the mean value in the remainder of Taylor's theorem.

Observe that

$$
\int_{u}^{\hat{t}_{u, \sigma}^{\varepsilon}} \mathbf{F}^{\varepsilon}\left(\hat{\mathbf{x}}_{u, \sigma}^{\varepsilon}\left(t^{\prime}\right)\right) d t^{\prime}
$$

is $\mathscr{F}_{u}$-measurable and continuous in $u$.

The other terms of the expansion containing $\mathbf{x}_{u, \sigma}^{\varepsilon}\left(t^{\prime}\right)$ are still anticipative and we need to expand them further. Before doing this however we discuss the magnitude of the terms we have so far.

By the overlap lemma (4.1) we need only consider the term

on

$$
\int_{S \wedge \tau^{\varepsilon} S}^{t \wedge \tau^{\varepsilon}} N^{\varepsilon}(d \boldsymbol{\sigma} \cdot d u) \int_{u}^{t_{u, \sigma}} \mathbf{F}^{\varepsilon}\left(\mathbf{x}_{u, \sigma}^{\varepsilon}\left(t^{\prime}\right)\right) d t^{\prime}
$$

$$
\Lambda_{n}^{\varepsilon}=\left\{\sup _{s \leqq T} N_{n}^{\varepsilon}(s) \leqq \lambda\right\},
$$

where we pick $n$ large enough $(n \sim n(\phi, a))$, and where we allow for $\lambda$ to increase with an inverse power of $\varepsilon$ less than 1 .

Note that by (4.13)

$$
\sup _{u \leqq \tau^{\varepsilon}}\left|\hat{t}_{u, \sigma}^{\varepsilon}-u\right| \leqq 4 \varepsilon^{2}
$$

Furthermore it follows from this and the crossing time lemma (4.3) that there exists 
a constant $C \geqq 4$ such that on $\Lambda_{n}^{\varepsilon}$,

$$
\sup _{u \leqq t^{\prime} \leqq \hat{t}_{u, \sigma}^{\varepsilon}}\left|\mathbf{x}_{u, \sigma}^{\varepsilon}\left(t^{\prime}\right)-\hat{\mathbf{x}}_{u, \sigma}^{\varepsilon}\left(t^{\prime}\right)\right| \leqq C \lambda \varepsilon^{3}
$$

Introducing the expansion (4.15) into (4.16) we may now estimate some terms by summing absolute values on $\Lambda_{n}^{\varepsilon}$. For the remainder in the Taylor expansion we have on $\Lambda_{n}^{\varepsilon}$ by (4.17) and (4.18) that

$$
\begin{aligned}
& \sup _{s, t \leqq T}\left|\int_{S_{S \wedge \tau^{\varepsilon}}}^{t \wedge \tau^{\varepsilon}} N^{\varepsilon}(d \boldsymbol{\sigma}, d u) \int_{u}^{\hat{t}_{u, \sigma}^{\varepsilon}} \frac{1}{2}\left(\left(\mathbf{x}_{u, \sigma}^{\varepsilon}\left(t^{\prime}\right)-\hat{\mathbf{x}}_{u, \sigma}^{\varepsilon}\left(t^{\prime}\right)\right) \cdot \nabla\right)^{2} \mathbf{F}^{\varepsilon}\left(\mathbf{y}^{\varepsilon}\left(u, \boldsymbol{\sigma}, t^{\prime}\right)\right) d t^{\prime}\right| \\
& \quad \leqq N^{\varepsilon}(T) C^{2} \lambda^{2} \varepsilon^{6} B^{\prime \prime} \varepsilon^{-5} C \varepsilon^{2}=C^{3} \lambda^{2} \varepsilon^{3} N^{\varepsilon}(T) B^{\prime \prime},
\end{aligned}
$$

where

and

$$
B^{\prime \prime}=\sup _{\mathbf{e}_{1}, \mathbf{e}_{2}}\left|\mathbf{e}_{1} \cdot \nabla \mathbf{e}_{2} \cdot \nabla \mathbf{F}\right|
$$

$$
N^{\varepsilon}(T)=\int_{S}^{T} \int_{0}^{\tau^{\varepsilon}} N^{\varepsilon}(d \boldsymbol{\sigma}, d u)
$$

But by (3.6)

$$
\begin{aligned}
P^{\varepsilon}\left(\left\{N^{\varepsilon}(T)>\lambda / \varepsilon^{2}\right\}\right) & \leqq \varepsilon^{2} E\left(N^{\varepsilon}(T)\right) / \lambda=\frac{\varepsilon^{2}}{\lambda} E\left(\int_{S} \int_{0}^{T \wedge \tau^{\varepsilon}} \rho^{\varepsilon}(d \boldsymbol{\sigma}, d u)\right) \\
& \leqq \frac{1}{\lambda} \rho E\left(\int_{0}^{T \wedge \tau^{\varepsilon}} \int_{\left(v_{u}^{\varepsilon} \cdot \boldsymbol{\sigma}\right) \leqq 0}-\left(\mathbf{v}_{u}^{\varepsilon} \cdot d \boldsymbol{\sigma}\right) d u\right)=\frac{1}{\lambda} \rho \frac{3}{2} \pi T
\end{aligned}
$$

by definition of $\tau^{\varepsilon}$. (The reader should note that the second inequality would be an equality if there were no selfcrossings. In areas of selfcrossings $\rho^{\varepsilon}(d \boldsymbol{\sigma}, d u)$ is zero.)

We may now choose $\lambda=\varepsilon^{-\alpha}, \alpha<1 / 3$ to see that the "sup" above goes in probability to zero as $\varepsilon \rightarrow 0$.

The third term on the right of (4.15) yields

$$
\sup _{s \leqq t \leqq T}\left|\int_{S} \int_{S \wedge \tau^{\varepsilon}}^{t \wedge \tau^{\varepsilon}} N^{\varepsilon}(d \boldsymbol{\sigma}, d u) \int_{\hat{t}_{u, \sigma}^{\varepsilon}}^{t_{u, \sigma}^{\varepsilon}} \mathbf{F}^{\varepsilon}\left(\mathbf{x}_{u, \sigma}^{\varepsilon}\left(t^{\prime}\right)\right) d t^{\prime}\right| .
$$

To estimate this observe first that on $\Lambda_{n}^{\varepsilon}$ uniformly in $u$

and that

$$
\left|\mathbf{x}_{u, \sigma}^{\varepsilon}\left(t_{u, \sigma}^{\varepsilon}\right)-\hat{\mathbf{x}}_{u, \sigma}^{\varepsilon}\left(\hat{t}_{u, \sigma}^{\varepsilon}\right)\right| \leqq C \lambda \varepsilon^{3}
$$

$$
t_{u, \sigma}^{\varepsilon}-u<C \varepsilon^{2}
$$

and thus that for some constant $C^{\prime}$

$$
\sup _{u \leqq t \leqq t_{u, \sigma}^{\varepsilon}}\left|\mathbf{v}_{t}^{\varepsilon}-\mathbf{v}_{u}^{\varepsilon}\right|<C^{\prime} \lambda \varepsilon
$$

by the crossing time lemma (4.3)

Combining these facts one easily gets that for some constant $C^{\prime \prime}$,

so that

$$
\left|t_{u, \sigma}^{\varepsilon}-\hat{t}_{u, \sigma}^{\varepsilon}\right|<C^{\prime \prime} \lambda \varepsilon^{3}
$$

$$
\sup _{\hat{t}_{u, \sigma}^{\varepsilon} \leqq t \leqq t_{u, \sigma}^{\varepsilon}}\left|\mathbf{x}_{u, \sigma}^{\varepsilon}(t)-\hat{\mathbf{x}}_{u, \sigma}^{\varepsilon}\left(\hat{t}_{u, \sigma}^{\varepsilon}\right)\right|<C^{\prime \prime} \lambda \varepsilon^{3}
$$


But

$$
\sup _{\mathbf{y} \in\left\{\mathbf{x} ;\left|\mathbf{x}-\hat{c} S^{\varepsilon}\right|<C^{\prime \prime} \lambda \varepsilon^{3}\right\}}\left|\mathbf{F}^{\varepsilon}(\mathbf{y})\right|=B^{\prime} C^{\prime \prime} \lambda,
$$

and thus on $\Lambda_{n}^{\varepsilon}$ the "sup" above is bounded by $N^{\varepsilon}(T) C^{\prime 2} B^{\prime} \lambda^{2} \varepsilon^{3}$, and we may conclude as before that this term vanishes in probability as $\varepsilon \rightarrow 0$.

We consider now the first order term in (4.15):

$$
\int_{S}^{t} \int_{\wedge \wedge \tau^{\varepsilon}}^{t \wedge \tau^{\varepsilon}} N^{\varepsilon}(d \boldsymbol{\sigma}, d u) \int_{u}^{\hat{t}_{u, \sigma}^{\varepsilon}} d t^{\prime}\left(\mathbf{x}_{u, \sigma}^{\varepsilon}\left(t^{\prime}\right)-\hat{\mathbf{x}}_{u, \sigma}^{\varepsilon}\left(t^{\prime}\right)\right) \cdot \nabla \mathbf{F}^{\varepsilon}\left(\hat{\mathbf{x}}_{u, \sigma}^{\varepsilon}\left(t^{\prime}\right)\right) .
$$

A rough estimate gives that (4.19) $\sim \lambda$ on $\Lambda_{n}^{t}$.

We write

$$
\mathbf{X}_{u, \sigma}^{\varepsilon}\left(t^{\prime}\right)-\hat{\mathbf{x}}_{u, \sigma}^{\varepsilon}\left(t^{\prime}\right)=\int_{u}^{t^{\prime}} d t^{\prime \prime}\left(\mathbf{v}_{t^{\prime \prime}}^{\varepsilon}-\mathbf{v}_{u}^{\varepsilon}\right)
$$

and by $(3.10)$

$$
\begin{aligned}
\mathbf{x}_{u, \sigma}^{\varepsilon}\left(t^{\prime}\right)-\hat{\mathbf{x}}_{u, \sigma}^{\varepsilon}\left(t^{\prime}\right)= & \int_{u}^{t^{\prime}} d t^{\prime \prime}\left\{\int_{u}^{t^{\prime \prime}} \int_{S} N^{\varepsilon}\left(d \boldsymbol{\sigma}^{\prime}, d u^{\prime}\right) \int_{u^{\prime}}^{t^{\prime \prime}} \mathbf{F}^{\varepsilon}\left(\mathbf{x}_{u^{\prime}, \sigma^{\prime}}^{\varepsilon}\left(t^{\prime \prime \prime}\right)\right) d t^{\prime \prime \prime}\right. \\
& +\int_{0}^{u} \int_{S} N^{\varepsilon}\left(d \boldsymbol{\sigma}^{\prime}, d u^{\prime}\right) \int_{u}^{t^{\prime \prime}} \mathbf{F}^{\varepsilon}\left(\mathbf{x}_{u^{\prime}, \sigma^{\prime}}^{\varepsilon}\left(t^{\prime \prime \prime}\right)\right) d t^{\prime \prime \prime} \\
& \left.+\Delta \mathbf{v}_{0}^{\varepsilon}\left(t^{\prime \prime}\right)-\Delta \mathbf{v}_{0}^{\varepsilon}(u)\right\}
\end{aligned}
$$

We insert this in (4.19) and obtain

$$
\begin{gathered}
\int_{S} \int_{\wedge}^{t \wedge \tau^{\varepsilon}} N^{\varepsilon}(d \boldsymbol{\sigma}, d u) \int_{u}^{t_{u, \sigma}^{\varepsilon}} d t^{\prime} \int_{u}^{t^{\prime}} d t^{\prime \prime}\left\{\int_{u}^{t^{\prime \prime}} \int_{S} N^{\varepsilon}\left(d \boldsymbol{\sigma}^{\prime}, d u^{\prime}\right) \int_{u^{\prime}}^{t^{\prime \prime}} \mathbf{F}^{\varepsilon}\left(\mathbf{x}_{u^{\prime}, \sigma^{\prime}}^{\varepsilon}\left(t^{\prime \prime \prime}\right)\right) d t^{\prime \prime \prime}\right. \\
\left.+\int_{0}^{u} \int_{S} N^{\varepsilon}\left(d \boldsymbol{\sigma}^{\prime}, d u^{\prime}\right) \int_{u}^{t^{\prime \prime}} \mathbf{F}^{\varepsilon}\left(\mathbf{x}_{u^{\prime}, \sigma^{\prime}}^{\varepsilon}\left(t^{\prime \prime \prime}\right)\right) d t^{\prime \prime \prime}+\Delta \mathbf{v}_{0}^{\varepsilon}\left(t^{\prime \prime}\right)-\Delta \mathbf{v}_{0}^{\varepsilon}(u)\right\} .
\end{gathered}
$$

This is again in probability close to

$$
\begin{aligned}
& \int_{S}^{t} \int_{S \wedge \tau^{\varepsilon}}^{t \wedge \tau^{\varepsilon}} N^{\varepsilon}(d \boldsymbol{\sigma}, d u) \int_{u}^{\hat{t}_{u, \sigma}^{\varepsilon}} d t^{\prime} \int_{u}^{t^{\prime}} d t^{\prime \prime}\left\{\int_{u}^{t^{\prime \prime}} \int_{S} N^{\varepsilon}\left(d \boldsymbol{\sigma}^{\prime}, d u^{\prime}\right) \int_{u^{\prime}}^{t^{\prime \prime}} \mathbf{F}^{\varepsilon}\left(\hat{\mathbf{x}}_{u^{\prime}, \sigma^{\prime}}^{\varepsilon}\left(t^{\prime \prime \prime}\right)\right) d t^{\prime \prime \prime}\right. \\
& \left.+\int_{u-4 \varepsilon^{2}}^{u} \int_{S} N^{\varepsilon}\left(d \boldsymbol{\sigma}^{\prime}, d u^{\prime}\right) \int_{u}^{t^{\prime \prime}} \mathbf{F}^{\varepsilon}\left(\hat{\mathbf{x}}_{u^{\prime}, \sigma^{\prime}}^{\varepsilon}\left(t^{\prime \prime \prime}\right)\right) d t^{\prime \prime \prime}\right\},
\end{aligned}
$$

essentially repeating the estimates on $\Lambda_{n}^{\varepsilon}$ as before and noting that by (4.4),

$$
\begin{aligned}
& \int_{S}^{t \wedge} \int_{S \wedge \tau^{\varepsilon}}^{t \wedge} N^{\varepsilon}(d \boldsymbol{\sigma}, d u) \int_{u}^{t_{u, \sigma}^{\varepsilon}} d t^{\prime} \int_{u}^{t^{\prime \prime}} d t^{\prime \prime}\left\{\int_{0}^{u-4 \varepsilon^{2}} \int_{S} N^{\varepsilon}\left(d \boldsymbol{\sigma}^{\prime}, d u^{\prime}\right) \int_{u}^{t^{\prime \prime}} \mathbf{F}^{\varepsilon}\left(\mathbf{x}_{u^{\prime}, \sigma^{\prime}}^{\varepsilon}\left(t^{\prime \prime \prime}\right)\right) d t^{\prime \prime \prime}\right. \\
& \left.\quad+\Delta \mathbf{v}_{0}^{\varepsilon}\left(t^{\prime \prime}\right)-\Delta \mathbf{v}_{0}^{\varepsilon}(u)\right\}
\end{aligned}
$$

involves only scatterers which overlap areas of selfintersections or which overlap the particle initially. Hence we may estimate this term in a similar way as below (4.12). 
In view of (4.20) we set

$$
\begin{aligned}
\mathbf{y}_{+}^{\varepsilon}(t)-\mathbf{y}_{+}^{\varepsilon}(s)= & \int_{S}^{t \wedge} \int_{\wedge \tau^{\varepsilon}}^{t \wedge} \tau^{\varepsilon}(d \boldsymbol{\sigma}, d u) \int_{u}^{\hat{t}_{u, \sigma}^{\varepsilon}} d t^{\prime} \int_{u}^{t^{\prime}} d t^{\prime \prime} \int_{u}^{t^{\prime \prime}} \int_{S} N^{\varepsilon}\left(d \boldsymbol{\sigma}^{\prime}, d u^{\prime}\right) \\
& \cdot \int_{u^{\prime}}^{t \prime} d t^{\prime \prime \prime} \mathbf{F}^{\varepsilon}\left(\hat{\mathbf{x}}_{u^{\prime}, \sigma^{\prime}}^{\varepsilon}\left(t^{\prime \prime \prime}\right)\right) \cdot \nabla \mathbf{F}^{\varepsilon}\left(\hat{\mathbf{x}}_{u, \sigma}^{\varepsilon}\left(t^{\prime}\right)\right) \\
= & \int_{S}^{t \wedge \tau^{\tau^{\varepsilon}}} \int_{\tau^{\wedge} \tau^{\varepsilon}}^{\varepsilon} N^{\varepsilon}\left(d \boldsymbol{\sigma}^{\prime}, d u^{\prime}\right)\left\{\int_{\hat{t}_{u, \sigma}}^{u^{\prime}-} \tau^{\varepsilon} N^{\varepsilon}(d \boldsymbol{\sigma}, d u) \chi\left(\hat{t}_{u, \sigma}^{\varepsilon} \geqq u^{\prime}\right)\right. \\
& \left.\cdot \int_{u}^{t^{\prime}} d t^{\prime} \int_{u^{\prime}} d t^{\prime \prime} \int_{u^{\prime}}^{t^{\prime \prime}} d t^{\prime \prime \prime} \mathbf{F}^{\varepsilon}\left(\hat{\mathbf{x}}_{u^{\prime}, \sigma^{\prime}}^{\varepsilon}\left(t^{\prime \prime \prime}\right)\right) \cdot \nabla \mathbf{F}^{\varepsilon}\left(\hat{\mathbf{x}}_{u, \sigma}^{\varepsilon}\left(t^{\prime}\right)\right)\right\} \\
= & \int_{S_{\Delta}}^{t \wedge \tau^{\varepsilon} \tau^{\varepsilon}} \int^{\varepsilon} N^{\varepsilon}\left(d \boldsymbol{\sigma}^{\prime}, d u^{\prime}\right)\left\{\Delta \mathbf{v}_{+}^{\varepsilon}\left(u^{\prime}, \boldsymbol{\sigma}^{\prime}\right)\right\},
\end{aligned}
$$

where the second equality comes through a change in the order of integration. Note that $\mathbf{v}_{+}^{\varepsilon}\left(u^{\prime}, \boldsymbol{\sigma}^{\prime}\right)$ is as function of $u^{\prime}$ adapted and left continuous.

For the second summand in (4.20) we set

with

$$
\mathbf{y}_{-}^{\varepsilon}(t)-\mathbf{y}_{-}^{\varepsilon}(s)=\int_{S} \int_{S \wedge \tau^{\varepsilon}}^{t \wedge \tau^{\varepsilon}} N^{\varepsilon}(d \boldsymbol{\sigma}, d u) \Delta \mathbf{v}_{-}^{\varepsilon}(u, \boldsymbol{\sigma})
$$

$$
\begin{aligned}
\Delta \mathbf{v}_{-}^{\varepsilon}(u, \boldsymbol{\sigma})= & \int_{u}^{\hat{t}_{u, \sigma}^{\varepsilon}} d t^{\prime} \int_{u}^{t^{\prime}} d t^{\prime \prime} \int_{u-4 \varepsilon^{2}}^{u} \int_{S} N^{\varepsilon}\left(d \boldsymbol{\sigma}^{\prime}, d u^{\prime}\right) \\
& \cdot \int_{u}^{t^{\prime \prime}} d t^{\prime \prime \prime} \mathbf{F}^{\varepsilon}\left(\hat{\mathbf{x}}_{u^{\prime}, \sigma^{\prime}}^{\varepsilon}\left(t^{\prime \prime \prime}\right)\right) \cdot \nabla \mathbf{F}^{\varepsilon}\left(\hat{\mathbf{x}}_{u, \sigma}^{\varepsilon}\left(t^{\prime}\right)\right) \\
= & \tilde{\Delta} \mathbf{v}_{-}^{\varepsilon}(u, \boldsymbol{\sigma})+\int_{u}^{\hat{t}_{u, \sigma}^{\varepsilon}} d t^{\prime} \int_{u}^{t^{\prime}} d t^{\prime \prime} \Delta N^{\varepsilon}(u) \\
& \cdot \int_{u}^{t^{\prime \prime}} d t^{\prime \prime \prime} \mathbf{F}^{\varepsilon}\left(\hat{\mathbf{x}}_{u, \sigma}^{\varepsilon}\left(t^{\prime \prime \prime}\right)\right) \cdot \nabla \mathbf{F}^{\varepsilon}\left(\hat{\mathbf{x}}_{u, \sigma}^{\varepsilon}\left(t^{\prime}\right)\right),
\end{aligned}
$$

where in the last equality we split $\Delta \mathbf{v}_{-}^{\varepsilon}(u, \sigma)$ into its adapted left continuous part $\Delta \tilde{\mathbf{v}}_{-}^{\varepsilon}(u, \sigma)$ and the "jump at $u$ ", with

$$
\Delta N^{\varepsilon}(u)=N^{\varepsilon}(S,[0, u])-N^{\varepsilon}(S,[0, u]) .
$$

We are thus led to consider (4.10) for the "processes" $\mathbf{y}_{+}^{\varepsilon}(t), \mathbf{y}_{-}(t)$ and

$$
\mathbf{y}_{0}^{\varepsilon}(t)=\int_{S}^{t \wedge} \int_{0}^{\tau^{\varepsilon}} N^{\varepsilon}(d \boldsymbol{\sigma}, d u) \int_{u}^{t_{u, \sigma}^{\varepsilon}} d t^{\prime} \mathbf{F}^{\varepsilon}\left(\hat{\mathbf{x}}_{u, \sigma}^{\varepsilon}\left(t^{\prime}\right)\right),
$$

the last one representing the zeroth order term of the expansion (4.15), since we showed that up to terms which vanish in probability when $\varepsilon \rightarrow 0(\sim)$,

$$
\mathbf{v}_{t}^{\varepsilon} \sim \mathbf{y}_{+}^{\varepsilon}(t)+\mathbf{y}_{-}^{\varepsilon}(t)+\mathbf{y}_{0}^{\varepsilon}(t)
$$

We shall use the following [11]

(4.25) (Tightness Criterion) Lemma. Suppose that for all $\varepsilon>0$ there exists $\delta_{\varepsilon}>0$ such that 
(i) there exist $\beta>0, \zeta>1$ and $C>0$ such that for all $\varepsilon$ sufficiently small,

$$
E^{\varepsilon}\left(\left|\mathbf{y}_{+,-}^{\varepsilon}(t)-\mathbf{y}_{+,-}^{\varepsilon}(s)\right|^{\beta}\right) \leqq C|t-s|^{\zeta}
$$

for all $s, t \leqq T$ with $|t-s|<\delta_{\varepsilon}$, and

(ii) for every $\gamma>0$ and $\eta>0$ and $\varepsilon$ sufficiently small

$$
P^{\varepsilon}\left(\left\{\sup _{\substack{s, t \leq T \\|s-t|<\delta_{\varepsilon}}}\left|\mathbf{y}_{+,-}^{\varepsilon}(t)-\mathbf{y}_{+,--}^{\varepsilon}(s)\right|>\gamma\right\}\right)<\eta .
$$

Then for every $\gamma>0$ and $\eta>0$ there exists $\delta>0$ such that for all $\varepsilon$ sufficiently small

$$
P\left(\left\{\sup _{\substack{s, t \leq T \\|s-t|<\delta}}\left|\mathbf{y}_{+,-}^{\varepsilon}(t)-\mathbf{y}_{+,-}^{\varepsilon}(s)\right|>\gamma\right\}\right)>\eta .
$$

Choosing $\delta_{\varepsilon}=\varepsilon^{2}$ (4.27) is almost trivial by the overlap lemma (4.1). In fact on $\Lambda_{n}^{\varepsilon}$ it is easily seen that there exists a constant $C$ such that

$$
\sup _{\substack{s, t \leqq T \\|s-t|<\delta_{\varepsilon}}}\left|\mathbf{y}_{+,-}^{\varepsilon}(t)-\mathbf{y}_{+,-}^{\varepsilon}(s)\right| \leqq C \sup _{\substack{i \leqq T \wedge \tau^{t^{*}} \\ \mid v^{2}}} N^{\varepsilon}\left(S,\left[s, s+\delta_{\varepsilon}\right]\right) \lambda \varepsilon^{2},
$$

and since $v_{t}^{\varepsilon} \leqq \frac{3}{2}$, the particle cannot cover more area in time $\delta_{\varepsilon}$ than a ball of radius $\frac{3}{2} \delta_{\varepsilon}$. Hence appealing to the overlap lemma we find another constant $C^{\prime}$ such that on $\Lambda_{n}^{\varepsilon}$ the "sup" above is bounded by $C^{\prime} \lambda^{2} \varepsilon^{2}$ which goes to zero for the allowed values of $\lambda$.

We establish now (4.26) for $\delta_{\varepsilon}=\varepsilon^{2}$ and $\beta=2$, starting with $\mathbf{y}_{-}^{\varepsilon}$. Observing (4.22) and (4.23), we obtain

$$
\begin{aligned}
& E^{\varepsilon}\left(\left|\mathbf{y}_{-}^{\varepsilon}(t)-\mathbf{y}_{-}^{\varepsilon}(s)\right|^{2}\right)=E^{\varepsilon}\left(\left\{\int_{S} \int_{S \wedge \tau^{\varepsilon}}^{t \wedge \tau^{\varepsilon}} N^{\varepsilon}(d \boldsymbol{\sigma}, d u) \tilde{\Delta} \mathbf{v}_{-}^{\varepsilon}(u, \boldsymbol{\sigma})\right.\right. \\
& +\int_{S}^{t \wedge} \int_{S \wedge \tau^{\varepsilon}}^{\tau^{\varepsilon}} N^{\varepsilon}(d \boldsymbol{\sigma}, d u) \int_{u}^{\hat{t}_{u, \sigma}^{\varepsilon}} d t^{\prime} \int_{u}^{t^{\prime}} d t^{\prime \prime} \int_{u}^{t^{\prime \prime}} d t^{\prime \prime \prime} \mathbf{F}^{\varepsilon}\left(\hat{\mathbf{x}}_{u, \sigma}^{\varepsilon}\left(t^{\prime \prime \prime}\right)\right) \\
& \left.\left.\cdot \nabla \mathbf{F}^{\varepsilon}\left(\hat{\mathbf{x}}_{u, \sigma}^{\varepsilon}\left(t^{\prime}\right)\right)\right\}^{2}\right) \leqq 4\left[E^{\varepsilon}\left(\left\{\int_{S} \int_{s \wedge \tau^{\varepsilon}}^{t \wedge \tau^{\varepsilon}} \rho^{\varepsilon}(d \boldsymbol{\sigma}, d u) \Delta \tilde{\mathbf{v}}_{-}^{\varepsilon}(u, \boldsymbol{\sigma})\right\}^{2}\right)\right. \\
& +E^{\varepsilon}\left(\left\{\int_{S} \int_{s \wedge \tau^{\varepsilon}}^{t \wedge \tau^{\varepsilon}} m^{\varepsilon}(d \boldsymbol{\sigma}, d u) \Delta \tilde{\mathbf{v}}_{-}^{\varepsilon}(u, \boldsymbol{\sigma})\right\}^{2}\right) \\
& +E^{\varepsilon}\left(\left\{\int_{S} \int_{S \wedge \tau^{\varepsilon}}^{t \wedge \tau^{\varepsilon}} \rho^{\varepsilon}(d \boldsymbol{\sigma}, d u) \int_{u}^{\hat{t}_{u, \sigma}^{\varepsilon}} d t^{\prime} \int_{u}^{t^{\prime}} d t^{\prime \prime} \int_{u}^{t^{\prime \prime}} d t^{\prime \prime \prime}\right.\right. \\
& \left.\left.\cdot \mathbf{F}^{\varepsilon}\left(\hat{\mathbf{x}}_{u, \sigma}^{\varepsilon}\left(t^{\prime \prime \prime}\right)\right) \cdot \nabla \mathbf{F}^{\varepsilon}\left(\hat{\mathbf{x}}_{u, \sigma}^{\varepsilon}\left(t^{\prime}\right)\right)\right\}^{2}\right) \\
& +E^{\varepsilon}\left(\left\{\int_{S} \int_{s \wedge \tau^{\varepsilon}}^{t \wedge \tau^{\varepsilon}} m^{\varepsilon}(d \boldsymbol{\sigma}, d u) \int_{u}^{t_{u, \sigma}^{\varepsilon}} d t^{\prime} \int_{u}^{t^{\prime}} d t^{\prime \prime} \int_{u}^{t^{\prime \prime}} d t^{\prime \prime \prime}\right.\right. \\
& \left.\left.\left.\cdot \mathbf{F}^{\varepsilon}\left(\hat{\mathbf{x}}_{u, \sigma}^{\varepsilon}\left(t^{\prime \prime \prime}\right)\right) \cdot \nabla \mathbf{F}^{\varepsilon}\left(\hat{\mathbf{x}}_{u, \sigma}^{\varepsilon}\left(t^{\prime}\right)\right)\right\}^{2}\right)\right] \text {, }
\end{aligned}
$$

where we used the splitting (3.6) and a binomial inequality. Furthermore the martingale measures integrate continuous $\widetilde{F}_{u}$-measurable functions so that (3.6a) 
holds. We may therefore apply Burkholder--Davis-Gundy inequalities [8], or, since we only take the square, the quadratic variation formula which yields for example that

$$
\begin{aligned}
& E^{\varepsilon}\left(\left\{\int_{S_{\varsigma} \wedge \tau^{\varepsilon}}^{t \wedge \tau^{\varepsilon}} m^{\varepsilon}(d \boldsymbol{\sigma}, d u) \tilde{\Delta} \mathbf{v}_{-}^{\varepsilon}(u, \boldsymbol{\sigma})\right\}^{2}\right) \\
& =E^{\varepsilon}\left(\left\{\int_{S_{S} \wedge \tau^{\varepsilon}}^{t \wedge \tau^{\varepsilon}} \rho^{\varepsilon}(d \boldsymbol{\sigma}, d u) \tilde{\Delta} \mathbf{v}_{-}^{\varepsilon}(u, \sigma)\right\}^{2}\right) \\
& =E^{\varepsilon}\left(\left\{\int_{S}^{t \wedge \tau_{s} \int^{\varepsilon}} \rho^{\varepsilon} \tau^{\varepsilon}(d \boldsymbol{\sigma}, d u) \Delta \mathbf{v}_{-}^{\varepsilon}(u, \boldsymbol{\sigma})\right\}^{2}\right),
\end{aligned}
$$

where the last equality comes from the continuity of the measure $\rho^{\varepsilon}$.

Before time $\tau^{\varepsilon}$ we have a simple bound on the rates (cf. below (4.18))

$$
\rho^{\varepsilon}(d \boldsymbol{\sigma}, d u) \leqq \frac{3}{2} \rho^{\varepsilon} \varepsilon^{2} d \sigma d u=\frac{3}{2} \rho \varepsilon^{-2} d \sigma d u, \quad \sigma \in[0, \pi) .
$$

Let $\tilde{N}^{\varepsilon}$ be the Poisson number of points with the density

$$
\frac{3}{2} \pi \rho \varepsilon^{-2} d u \text {. }
$$

Clearly, $\tilde{N}^{\varepsilon}$ is a Poisson domination of $N^{\varepsilon}$ for example in the sense of correlation functions (see e.g. $|9|$ ).

In view of (4.23) we easily estimate with (4.17), and observing the Poisson domination that, for example

$$
\begin{aligned}
E^{\varepsilon}\left(\Delta \mathbf{v}_{-}^{\varepsilon}(u, \sigma) \Delta \mathbf{v}_{-}^{\varepsilon}\left(u^{\prime}, \sigma^{\prime}\right)\right) & \leqq C \varepsilon^{4} E\left(\tilde{N}^{\varepsilon}\left(\left[u-4 \varepsilon^{2}, u+4 \varepsilon^{2}\right]\right) \tilde{N}^{\varepsilon}\left(\left[u^{\prime}-4 \varepsilon^{2}, u^{\prime}+4 \varepsilon^{2}\right]\right)\right. \\
& \leqq C^{\prime} \varepsilon^{4}
\end{aligned}
$$

where $C, C^{\prime}$ are appropriate constants, the latter coming from Schwartz's inequality and translation invariance.

Thus for the first term on the right of (4.28) we easily get that

$$
E^{\varepsilon}\left(\left\{\int_{S} \int_{\wedge}^{t \wedge \tau^{\varepsilon}} \rho^{\varepsilon}(d \boldsymbol{\sigma}, d u) \Delta \mathbf{v}_{-}^{\varepsilon}(u, \boldsymbol{\sigma})\right\}^{2}\right) \leqq C^{\prime \prime}|t-s|^{2}
$$

for some constant $C^{\prime \prime}$.

Equation (4.29) and all the other terms may be handled the same way, producing $\zeta=2$ in (4.26) for $|t-s| \geqq \varepsilon^{2}$.

Next we observe that $\Delta \mathbf{v}_{+}^{\varepsilon}$ is by (4.17) essentially the same as $\Delta \mathbf{v}_{-}^{\varepsilon}$, and to show (4.26) for $\mathbf{y}_{+}^{\varepsilon}$ is therefore only a repetition of the foregoing without the extra term coming from the "jump at $u$."

Therefore the conclusion of Lemma (4.25) holds, and hence we are left with showing (4.10) for $\mathbb{y}_{0}^{\varepsilon}$ (cf. (4.24)). For this let

$$
T_{i}^{\varepsilon}, \widetilde{T}_{i}^{\varepsilon}, i=1, \ldots, K, T_{i}^{\varepsilon}, \widetilde{T}_{i}^{\varepsilon}<\tau^{\varepsilon}
$$

denote the first entrance and exit time of the particle of the tube $T_{t}^{\varepsilon}, t \leqq T$, at the $i$-th selfcrossing. We define a new point process $\hat{N}^{\varepsilon}(d \sigma, d u)$ by

$$
\begin{aligned}
\hat{N}^{\varepsilon}(d \boldsymbol{\sigma}, d u) & =N^{\varepsilon}(d \boldsymbol{\sigma}, d u) \\
\text { on the set } N S C & =\left[0, T_{1}^{\varepsilon}\right] \cup\left[\tilde{T}_{1}^{\varepsilon}, T_{2}^{\varepsilon}\right] \cup \cdots
\end{aligned}
$$


and on $[0, T]-N S C, \hat{N}^{\varepsilon}(d \boldsymbol{\sigma}, d u)$ is a Poisson point measure with intensity

$$
\hat{\rho}^{\varepsilon}(d \boldsymbol{\sigma}, d u)=-\rho \varepsilon^{2} \mathbf{v}_{u}^{\varepsilon} \cdot d \boldsymbol{\sigma} d u \vee 0 .
$$

We denote the appropriate probability space by $\left(\hat{\Omega}, \hat{\mathscr{F}}, \hat{P}^{\varepsilon}\right)\left(\hat{\mathscr{F}}_{t} \uparrow \hat{\mathscr{F}}\right)$.

By the argument on the effect of selfcrossings following (4.12) we need only show (4.10) for

$$
\hat{y}_{0}^{\varepsilon}(t)=\int_{S}^{t \wedge} \int_{\wedge \tau^{\varepsilon}}^{t \tau^{\varepsilon}} \widehat{N}^{\varepsilon}(d \boldsymbol{\sigma}, d u) \int_{u}^{\hat{t}_{u, \sigma}^{\varepsilon}} \mathbf{F}^{\varepsilon}\left(\hat{x}_{u, \sigma}^{\varepsilon}\left(t^{\prime}\right)\right) d t^{\prime} .
$$

We shall obtain this by showing (4.26) for $\hat{\mathbf{y}}_{0}^{\varepsilon}, t-s \geqq \varepsilon^{2}$. (4.27) follows then almost verbatim as before.

Since the compensator of $\hat{N}^{\varepsilon}(d \boldsymbol{\sigma}, d u)$ is given in (4.32), we easily have that by rotational symmetry

$$
\int_{S} \hat{\rho}^{\varepsilon}(d \boldsymbol{\sigma}, d u) \int_{u}^{\hat{t}_{u, \sigma}^{\varepsilon}} \mathbf{F}^{\varepsilon}\left(\hat{\mathbf{x}}_{u, \sigma}^{\varepsilon}\left(t^{\prime}\right)\right) d t^{\prime}=0 .
$$

Hence for $\gamma>0$, by the splitting (3.6) applied to $\widehat{N}^{\varepsilon}$ and by (4.34),

$$
\begin{aligned}
& \hat{E}^{\varepsilon}\left(\left|\mathbf{y}_{0}^{\varepsilon}(t)-\mathbf{y}_{0}^{\varepsilon}(s)\right|^{2+\gamma}\right)=\hat{E}^{\varepsilon}\left(\left|\int_{S} \int_{\lrcorner \wedge \tau^{\varepsilon}}^{t \wedge \tau^{\varepsilon}} \hat{N}^{\varepsilon}(d \boldsymbol{\sigma}, d u) \int_{u}^{\hat{t}_{u, \sigma}^{\varepsilon}} d t^{\prime} \mathbf{F}^{\varepsilon}\left(\hat{\mathbf{x}}_{u, \sigma}^{\varepsilon}\left(t^{\prime}\right)\right)\right|^{2+\gamma}\right) \\
& =\hat{E}^{\varepsilon}\left(\left|\int_{S_{S}}^{t \wedge \tau^{\varepsilon}} \int_{\tau^{\varepsilon}}^{\varepsilon} \hat{m}^{\varepsilon}(d \boldsymbol{\sigma}, d u) \int_{u}^{\hat{t}_{u, \sigma}^{\varepsilon}} d t^{\prime} \mathbf{F}^{\varepsilon}\left(\hat{\mathbf{x}}_{u, \sigma}^{\varepsilon}\left(t^{\prime}\right)\right)\right|^{2+\gamma}\right) \\
& \leqq C \widehat{E}^{\varepsilon}\left(\left|\int_{S} \int_{S \wedge \tau^{\varepsilon}}^{t \wedge \tau^{\varepsilon}} \hat{\rho}^{\varepsilon}(d \boldsymbol{\sigma}, d u)\left(\int_{u}^{t_{u, \sigma}^{\varepsilon}} d t^{\prime} \mathbf{F}^{\varepsilon}\left(\hat{\mathbf{x}}_{u, \sigma}^{\varepsilon}\left(t^{\prime}\right)\right) d t^{\prime}\right)^{2}\right|^{2+\gamma / 2}\right) \\
& \leqq C^{\prime}|t-s|^{1+\gamma / 2} \text {, }
\end{aligned}
$$

where we used the Burkholder-Davis-Gundy inequality on the martingale (note that $\hat{m}^{\varepsilon}$ integrates a continuous $\hat{\mathscr{F}}_{u}$-measurable function) and for the last inequality the bound (4.30) with (4.17) and $t-s \geqq \varepsilon^{2}$. $C$ and $C^{\prime}$ are constants depending on $\gamma$ and $B^{\prime}$.

Thus Proposition (4.9) holds!

\section{The Martingale Problem}

We established tightness of the family $\left(\tilde{v}^{\varepsilon}\right)_{\varepsilon>0}$. Every limit point of subsequences $\left(\tilde{v}^{\varepsilon_{n}}\right)_{\varepsilon_{n}}$ has support in $C[0, T]^{2}$. We obtain uniqueness by showing that all limit points satisfy the martingale problem associated with the diffusion process $\mathbf{v}_{t}$ given in Theorem (1.3). It is sufficient to establish the martingale problem for the functions $\mathbf{p}$ and $\mathbf{p} \mathbf{p}|4|$.

(5.1) Proposition (Martingale Problem). Let $L$ be as in (1.6). For every $s, t \in[0, T]$ and every smooth and bounded cylinder function $\phi_{s}$ depending only on $\mathbf{p}(u), u \leqq s$, $\mathbf{p} \in C[0, T]^{2}$ we have that for $\mathbf{f}=\mathbf{p}$ and $\mathbf{f}=\mathbf{p} \mathbf{p}$,

$$
\lim _{\varepsilon \rightarrow 0} \tilde{v}^{\varepsilon}\left(\left\{\mathbf{f}(\mathbf{p}(t))-\mathbf{f}(\mathbf{p}(s))-\int_{s \wedge \tau}^{t \wedge \tau} L \mathbf{f}(\mathbf{p}(u)) d u\right\} \Phi_{s}\right)=0 .
$$


Proof. By (1.6)

$$
\begin{gathered}
L \mathbf{p}=\pi \rho \nabla_{p} \cdot \int d^{2} \mathbf{k} \mathbf{k} \mathbf{k} \delta(\mathbf{k} \cdot \mathbf{p})|\hat{V}(k)|^{2}, \\
L \mathbf{p} \mathbf{p}=2 \pi \rho \nabla_{p} \cdot \int d^{2} \mathbf{k} \mathbf{k} \mathbf{k} \delta(\mathbf{k} \cdot \mathbf{p})|\hat{V}(k)|^{2} \mathbf{p} .
\end{gathered}
$$

To show (5.1) for $\mathbf{f}=\mathbf{p}$ is equivalent to showing that

$$
\lim _{\varepsilon \rightarrow 0} E^{\varepsilon}\left(\left(\mathbf{v}_{t}^{\varepsilon}-\mathbf{v}_{s}^{\varepsilon}-\int_{s \wedge \tau^{\varepsilon}}^{t \wedge \tau^{\varepsilon}} L \mathbf{v}_{u}^{\varepsilon} d s\right) \Phi_{s}^{\varepsilon}\right)=0
$$

where $\Phi_{s}^{\varepsilon}$ is the function $\Phi$ at $\mathbf{p}=\mathbf{v}^{\varepsilon}$.

By (4.12)

$$
E^{\varepsilon}\left(\left(\mathbf{v}_{t}^{\varepsilon}-\mathbf{v}_{s}^{\varepsilon}\right) \Phi_{s}^{\varepsilon}\right)=E^{\varepsilon}\left(\left\{\int_{S}^{t \wedge} \int_{s \wedge \tau^{\varepsilon}}^{t \tau^{\varepsilon}} N^{\varepsilon}(d \boldsymbol{\sigma}, d u) \int_{u}^{t_{u, \sigma}^{\varepsilon}} d t^{\prime} \mathbf{F}^{\varepsilon}\left(\mathbf{x}_{u, \sigma}^{\varepsilon}\left(t^{\prime}\right)\right)\right\} \Phi\right)+o(1),
$$

where $o(1)$ accounts for the selfcrossing and overlap terms in (4.12): By the argument below (4.12) and the overlap lemma (4.3) we obtain easily that these terms are bounded in $L^{1}\left(d P^{\varepsilon}\right)$ by

$$
\begin{gathered}
C \lambda \varepsilon+C^{\prime} T \frac{1}{\varepsilon} E^{\varepsilon}\left(\sup _{s \leqq T} N_{n(\phi, a)}^{\varepsilon}(s) \chi\left(\sup _{s \leqq T} N_{n(\phi, a)}^{\varepsilon}(s)>\lambda\right)\right) \\
=C \lambda \varepsilon+C^{\prime} T \frac{1}{\varepsilon} \int_{\lambda}^{\infty} P^{\varepsilon}\left(\sup _{s \leqq T} N_{n(\phi, a)}^{\varepsilon}(s)>x\right) d x=o(1),
\end{gathered}
$$

where $C, C^{\prime}$ are appropriate constants depending on $\Phi, B^{\prime}$ and $n(\phi, a)$ and $\lambda \sim \varepsilon^{-a}$, $\alpha<1$.

Introducing now the expansion (4.15) into (5.5) we arrive in accordance with (4.24) by estimates very similar to (5.6) at

$$
E^{\varepsilon}\left(\left(\mathbf{v}_{t}^{\varepsilon}-\mathbf{v}_{s}^{\varepsilon}\right) \Phi_{s}^{\varepsilon}\right)=E^{\varepsilon}\left(\left(\mathbf{y}_{-}^{\varepsilon}(t)-\mathbf{y}_{-}^{\varepsilon}(s)+\mathbf{y}_{+}^{\varepsilon}(t)-\mathbf{v}_{+}^{\varepsilon}(s)-\hat{\mathbf{y}}_{0}^{\varepsilon}(t)-\hat{\mathbf{y}}_{0}^{\varepsilon}(s)\right) \Phi_{s}^{\varepsilon}\right)+o(1),
$$

where we substituted (4.21), (4.22) and already did the step (4.33). But by (4.34) and the martingale property

$$
E^{\varepsilon}\left(\left(\hat{\mathbf{y}}_{0}^{\varepsilon}(t)-\hat{\mathbf{y}}_{0}^{\varepsilon}(s)\right) \Phi_{s}^{\varepsilon}\right)=0 .
$$

Now define $\hat{\mathbf{y}}_{+,-}^{\varepsilon}$ by replacing everywhere $N^{\varepsilon}$ by $\hat{N}^{\varepsilon}$ in $\mathbf{y}_{+,-}$, which we can do at the cost of another error $o(1)$, essentially repeating the arguments of before. We shall therefore show that

$$
\lim _{\varepsilon \rightarrow 0} \hat{E}^{\varepsilon}\left(\Phi_{S}^{\varepsilon}\left(\int_{S}^{t \wedge} \int_{\wedge}^{t \tau^{\varepsilon}} \hat{N}^{\varepsilon}(d \boldsymbol{\sigma}, d u)\left\{\Delta \hat{\tilde{\mathbf{v}}}_{-}^{\varepsilon}(u, \boldsymbol{\sigma})+\Delta \hat{\mathbf{v}}_{+}(u, \boldsymbol{\sigma})\right\}\right)\right)=0
$$

and that

$$
\begin{aligned}
& \lim _{\varepsilon \rightarrow 0} \hat{E}^{\varepsilon}\left(\Phi _ { s } ^ { \varepsilon } \left(\int_{S} \int_{s \wedge \tau^{\varepsilon}}^{t \wedge \tau^{\varepsilon}} \hat{N}^{\varepsilon}(d \boldsymbol{\sigma}, d u) \int_{u}^{\hat{t}_{u, \sigma}^{\varepsilon}} d t^{\prime} \int_{u}^{t^{\prime}} d t^{\prime \prime} \int_{u}^{t^{\prime \prime}} d t^{\prime \prime \prime}\right.\right. \\
& \left.\left.\cdot \mathbf{F}^{\varepsilon}\left(\hat{\mathbf{x}}_{u, \sigma}^{\varepsilon}\left(t^{\prime \prime \prime}\right)\right) \cdot \nabla \mathbf{F}^{\varepsilon}\left(\hat{\mathbf{x}}_{u, \sigma}^{\varepsilon}\left(t^{\prime}\right)\right)-\int_{s \wedge \tau^{\varepsilon}}^{t \wedge \tau^{\varepsilon}} L \mathbf{v}_{u}^{\varepsilon} d u\right)\right)=0 .
\end{aligned}
$$

We start with (5.7). Recall that (in view of (4.21) and (4.23)) $\hat{N}^{\varepsilon}$ integrates in (5.7) only adapted left continuous processes; thus decomposing $\hat{N}^{\varepsilon}$ as in (3.6) the 
martingale part vanishes and we may replace $\hat{N}^{\varepsilon}$ by $\hat{\rho}^{\varepsilon}$. Next we change the order of integration in the term coming from $\hat{\mathbf{y}}_{+}^{\varepsilon}$ (corresponding to going in (4.21) backwards from the second to the first equality). We thus arrive at the following expression for the expectation in (5.7) (with an error $o(1)$ )

$$
\begin{aligned}
& \hat{E}^{\varepsilon}\left(\Phi _ { s } ^ { \varepsilon } \left\{\int_{S} \int_{S \wedge \tau^{\varepsilon}}^{t \wedge \tau^{\varepsilon}} \hat{\rho}^{\varepsilon}(d \boldsymbol{\sigma}, d u) \int_{u}^{t_{u, \sigma}^{\varepsilon}} d t^{\prime} \int_{u}^{t^{\prime}} d t^{\prime \prime} \int_{u-4 \varepsilon^{2}}^{u} \int_{S} \hat{N}^{\varepsilon}\left(d \boldsymbol{\sigma}^{\prime}, d u^{\prime}\right)\right.\right. \\
& \quad \cdot \int_{u}^{t^{\prime \prime}} \mathbf{F}^{\varepsilon}\left(\hat{\mathbf{x}}_{u^{\prime}, \sigma^{\prime}}^{\varepsilon}\left(t^{\prime \prime \prime}\right)\right) \cdot \nabla \mathbf{F}^{\varepsilon}\left(\hat{\mathbf{x}}_{u, \sigma}^{\varepsilon}\left(t^{\prime}\right)\right) d t^{\prime \prime \prime} \\
& +\int_{S_{S}} \int_{\wedge}^{t \wedge \tau^{\varepsilon}} \hat{N}^{\varepsilon} \hat{N}^{\varepsilon}(d \boldsymbol{\sigma}, d u) \int_{u}^{t_{u, \sigma}^{\varepsilon}} d t^{\prime} \int_{u}^{t^{\prime}} d t^{\prime \prime} \int_{u}^{t^{\prime \prime}} \int_{S} \hat{\rho}^{\varepsilon}\left(d \boldsymbol{\sigma}^{\prime}, d u^{\prime}\right) \\
& \left.\left.\quad \cdot \int_{u}^{t^{\prime \prime}} d t^{\prime \prime \prime} \mathbf{F}^{\varepsilon}\left(\hat{\mathbf{x}}_{u^{\prime}, \sigma^{\prime}}^{\varepsilon}\left(t^{\prime \prime \prime}\right)\right) \cdot \nabla \mathbf{F}^{\varepsilon}\left(\hat{\mathbf{x}}_{u, \sigma}^{\varepsilon}\left(t^{\prime}\right)\right)\right\}\right) .
\end{aligned}
$$

Let

$$
\hat{\rho}_{u}^{\varepsilon}\left(d \boldsymbol{\sigma}^{\prime}, d u^{\prime}\right)=-\boldsymbol{v}_{u}^{\varepsilon} \cdot d \boldsymbol{\sigma}^{\prime} \rho \varepsilon^{-2} d u^{\prime} \vee 0,
$$

and note that in variation norm on $\left[u, \hat{t}_{u, \sigma}^{\varepsilon}\right]$

Furthermore let

$$
\left\|\hat{\rho}_{u}^{\varepsilon}-\hat{\rho}^{\varepsilon}\right\|<\sup _{u \leqq u^{\prime} \leqq f_{u, \sigma}^{\varepsilon}}\left|\mathbf{v}_{u}^{\varepsilon}-\mathbf{v}_{u^{\prime}}^{\varepsilon}\right|
$$

$$
\hat{\mathbf{x}}_{u^{\prime}, \sigma^{\prime}, u}^{\varepsilon}(t)=\mathbf{v}_{u}^{\varepsilon}\left(t-u^{\prime}\right)+\boldsymbol{\sigma}^{\prime} \varepsilon^{2} .
$$

We replace now in the first expectation in $(5.1) \hat{\rho}^{\varepsilon}(d \boldsymbol{\sigma}, d u), \Phi_{s}^{\varepsilon}$ and $\nabla \mathbf{F}^{\varepsilon}\left(\hat{\mathbf{x}}_{u, \sigma}^{\varepsilon}\left(t^{\prime}\right)\right)$ by the "shifted" $\hat{\rho}_{u-4 \varepsilon^{2}}^{\varepsilon}(d \boldsymbol{\sigma}, d u), \Phi_{s-4 \varepsilon^{2}}^{\varepsilon}$ and $\nabla \mathbf{F}^{\varepsilon}\left(\hat{\mathbf{x}}_{u, \sigma, u-4 \varepsilon^{2}}^{\varepsilon}\left(t^{\prime}\right)\right)$, and again by estimates very similar to previous ones this produces an error $o(1)$. (For the $\nabla \mathbf{F}$ term recall the argument before (4.14).) But then $\hat{N}^{\varepsilon}\left(d \boldsymbol{\sigma}^{\prime}, d u^{\prime}\right)$ integrates only $\widehat{\mathscr{F}}_{u^{\prime}}$-measurable functions, so that from the decomposition of $\hat{N}^{\varepsilon}$ only $\hat{\rho}^{\varepsilon}\left(d \boldsymbol{\sigma}^{\prime}, d u^{\prime}\right)$ survives. We may then shift back again at the cost of $o(1)$.

In the second expectation in (5.9) we shift $\rho^{\varepsilon}\left(d \boldsymbol{\sigma}^{\prime}, d u^{\prime}\right)$ to $\hat{\rho}_{u}^{\varepsilon}\left(d \boldsymbol{\sigma}^{\prime}, d u^{\prime}\right)$ and $\mathbb{F}^{\varepsilon}\left(\hat{\mathbf{x}}_{u^{\prime}, \sigma^{\prime}}^{\varepsilon}\left(t^{\prime \prime \prime}\right)\right)$ to $\mathbf{F}^{\varepsilon}\left(\hat{\mathbf{x}}_{u^{\prime}, \sigma^{\prime}, u}^{\varepsilon}\left(t^{\prime \prime \prime}\right)\right)$ so that $\hat{N}^{\varepsilon}(d \boldsymbol{\sigma}, d u)$ integrates nonanticipative functions, and thus also here only the compensator part survives.

We thus come from (5.9) to

$$
\begin{aligned}
& \hat{E}\left(\Phi _ { s } ^ { \varepsilon } \left\{\int_{S} \int_{\varsigma \wedge \tau^{\varepsilon}}^{t \wedge \tau^{\varepsilon}} \hat{\rho}^{\varepsilon}(d \boldsymbol{\sigma}, d u) \int_{u}^{t_{u, \sigma}^{\varepsilon}} d t^{\prime} \int_{u}^{t^{\prime}} d t^{\prime \prime} \int_{u-4 \varepsilon^{2}}^{u} \int_{S} \hat{\rho}^{\varepsilon}\left(d \boldsymbol{\sigma}^{\prime}, d u^{\prime}\right)\right.\right. \\
& \left.\left.\quad \cdot \int_{u}^{t^{\prime \prime}} \mathbb{F}^{\varepsilon}\left(\hat{\mathbf{x}}_{u^{\prime}, \sigma^{\prime}}^{\varepsilon}\left(t^{\prime \prime \prime}\right)\right) \cdot \nabla \mathbb{F}^{\varepsilon}\left(\hat{\mathbf{x}}_{u, \sigma}^{\varepsilon}\left(t^{\prime}\right)\right) t^{\prime \prime \prime}\right\}\right) \\
& +\hat{E}^{\varepsilon}\left(\Phi _ { S } ^ { \varepsilon } \left\{\int_{S} \int_{s \wedge \tau^{\varepsilon}}^{t \wedge \tau^{\varepsilon}} \hat{\rho}^{\varepsilon}(d \boldsymbol{\sigma}, d u) \int_{u}^{t_{u, \sigma}^{\varepsilon}} d t^{\prime} \int_{u}^{t^{\prime}} d t^{\prime \prime} \int_{u}^{t^{\prime \prime}} \int_{S} \hat{\rho}^{\varepsilon}\left(d \boldsymbol{\sigma}^{\prime}, d u^{\prime}\right)\right.\right. \\
& \left.\left.\quad \cdot \int_{u^{\prime}}^{t^{\prime \prime}} \mathbf{F}^{\varepsilon}\left(\hat{\mathbf{x}}_{u^{\prime}, \sigma^{\prime}}^{\varepsilon}\left(t^{\prime \prime \prime}\right)\right) \cdot \nabla \mathbf{F}^{\varepsilon}\left(\hat{\mathbf{x}}_{u, \sigma}^{\varepsilon}\left(t^{\prime}\right)\right) d t^{\prime \prime \prime}\right\}\right)+o(1) .
\end{aligned}
$$

Note that the integral over the force in the second expectation above may as well 
start from $u$. Therefore combining the expressions in both expectations they become

$$
\begin{aligned}
& \int_{S} \int_{\wedge \wedge \tau^{\varepsilon}}^{t \wedge \tau^{\varepsilon}} \hat{\rho}^{\varepsilon}(d \boldsymbol{\sigma}, d u) \int_{u}^{t_{u, \sigma}^{\varepsilon}} d t^{\prime} \int_{u}^{t^{\prime}} d t^{\prime \prime} \int_{-\infty}^{t^{\prime \prime}} \hat{\rho}^{\varepsilon}\left(d \boldsymbol{\sigma}^{\prime}, d u^{\prime}\right) \\
& \cdot \int_{u}^{t^{\prime \prime}} d t^{\prime \prime \prime} \mathbf{F}^{\varepsilon}\left(\hat{\mathbf{x}}_{u^{\prime}, \sigma^{\prime}, u}^{\varepsilon}\left(t^{\prime \prime \prime}\right)\right) \cdot \nabla \mathbf{F}^{\varepsilon}\left(\hat{\mathbf{x}}_{u, \sigma}^{\varepsilon}\left(t^{\prime}\right)\right)+o(1) .
\end{aligned}
$$

Fixing all variables we do the integration over $u^{\prime}$ first: by (5.10) and (5.11)

$$
\begin{aligned}
\int_{-\infty}^{t^{\prime \prime}} d u^{\prime} \mathbf{F}^{\varepsilon}\left(\mathbf{v}_{u}^{\varepsilon}\left(t^{\prime \prime \prime}-u^{\prime}\right)+\boldsymbol{\sigma}^{\prime} \varepsilon^{2}\right) & =\int_{-\infty}^{t^{\prime \prime}} d u^{\prime} \mathbf{F}^{\varepsilon}\left(\mathbf{v}_{u}^{\varepsilon}\left(t^{\prime \prime \prime}-u^{\prime}\right)+\boldsymbol{\sigma}^{\prime} \varepsilon^{2}\right) \\
& =\varepsilon \int_{0}^{\infty} d w \mathbf{F}\left(\mathbf{v}_{u}^{\varepsilon} w+\boldsymbol{\sigma}^{\prime}\right)=0,
\end{aligned}
$$

since $\mathbf{F}$ is a gradient of a potential. (Note that if this were not the case the limit process would not live on the sphere, see [1].) Hence (5.12) is o(1), and (5.7) follows.

To show (5.8) is a matter of computation:

By (5.10), (4.13) and the martingale property we are led to consider

$$
\begin{aligned}
\hat{E}^{\varepsilon}\left(\Phi _ { s } ^ { \varepsilon } \left\{\int_{S} \int_{\wedge \wedge \tau^{\varepsilon}}^{t \wedge \tau^{\varepsilon}} \hat{\rho}^{\varepsilon}(d \boldsymbol{\sigma}, d u) \int_{u}^{t_{u, \sigma}^{\varepsilon}} d t^{\prime} \int_{u}^{t^{\prime}} d t^{\prime \prime} \int_{u}^{t^{\prime \prime}} d t^{\prime \prime \prime}\right.\right. \\
\left.\left.\cdot \mathbf{F}^{\varepsilon}\left(\hat{\mathbf{x}}_{u, \sigma}^{\varepsilon}\left(t^{\prime \prime \prime}\right)\right) \cdot \nabla \mathbf{F}^{\varepsilon}\left(\hat{\mathbf{x}}_{u, \sigma}^{\varepsilon}\left(t^{\prime}\right)\right)\right\}\right) \\
=E^{\varepsilon}\left(\Phi _ { s } ^ { \varepsilon } \left\{\int_{s \wedge \tau^{\varepsilon}}^{t \wedge \tau^{\varepsilon}} d u \frac{\rho}{\varepsilon^{2}} \int_{v_{u}^{\varepsilon} \sigma \geqq 0} \mathbf{v}_{u} \cdot d \boldsymbol{\sigma} \int_{u}^{t_{u, \sigma}^{\varepsilon}} d t^{\prime} \int_{u}^{t^{\prime}} d t^{\prime \prime} \int_{u}^{t^{\prime \prime}} d t^{\prime \prime \prime}\right.\right. \\
\left.\left.\quad \cdot \mathbf{F}\left(\mathbf{v}_{u}^{\varepsilon}\left(t^{\prime \prime \prime}-u\right) / \varepsilon^{2}+\boldsymbol{\sigma}\right) \cdot \frac{1}{\varepsilon^{3}} \nabla \mathbf{F}^{\varepsilon}\left(\mathbf{v}_{u}^{\varepsilon}\left(t^{\prime}-u\right) / \varepsilon^{2}+\boldsymbol{\sigma}\right)\right\}\right) .
\end{aligned}
$$

After routine manipulations one sees that the integrand of the $u$-integral may be written as

$$
\begin{aligned}
& \rho \int_{v_{u} \geqq 0} d \boldsymbol{\sigma} \int_{-\infty}^{\infty} d t^{\prime} \int_{-\infty}^{t^{\prime}} d t^{\prime \prime} \int_{-\infty}^{t^{\prime \prime}} d t^{\prime \prime \prime} \mathbf{F}\left(\mathbf{v}_{u}^{\varepsilon} t^{\prime \prime \prime}+\boldsymbol{\sigma}\right) \cdot \nabla \mathbf{F}\left(\mathbf{v}_{u}^{\varepsilon} t^{\prime}+\boldsymbol{\sigma}\right) \\
& =\rho \int_{\mathbf{v}_{u} \sigma \geqq 0} d \boldsymbol{\sigma} \int_{-\infty}^{\infty} d t^{\prime} \int_{-\infty}^{t^{\prime}} d t^{\prime \prime}\left(t^{\prime}-t^{\prime \prime}\right) \mathbf{F}\left(\mathbf{v}_{u}^{\varepsilon} t^{\prime \prime}+\boldsymbol{\sigma}\right) \cdot \nabla \mathbf{F}\left(\mathbf{v}_{u}^{\varepsilon} t^{\prime}+\boldsymbol{\sigma}\right) \\
& =-\rho \int d^{2} \mathbf{r} \int_{-\infty}^{0} d \tau \tau \mathbf{F}\left(\mathbf{r}+\mathbf{v}_{u}^{\varepsilon} \tau\right) \cdot \nabla \mathbf{F}(\mathbf{r})=\text { "by symmetry" } \\
& \cdot \frac{1}{2} \rho \int d^{2} \mathbf{r} \int_{-\infty}^{\infty} d \tau\left(\nabla_{\xi} \cdot \mathbf{F}(\mathbf{r}+\boldsymbol{\xi} \tau) \mathbf{F}(\mathbf{r})\right)_{\mid \xi=v_{u}^{\varepsilon}} \\
& =\frac{1}{2} \rho \nabla_{\xi} \cdot \int d^{2} \mathbf{k} \int_{-\infty}^{\infty} d \tau e^{\imath \mathbf{k} \cdot \xi \tau} \mathbf{k} \mathbf{k}|\hat{V}(k)|_{\mid \xi=\mathbf{v}_{u}^{\varepsilon}}^{2}
\end{aligned}
$$

by Plancherel's theorem. One finally observes that

$$
\frac{1}{2} \int_{-\infty}^{\infty} d \tau e^{i k} \xi_{\tau}=\pi \delta(\mathbf{k} \cdot \boldsymbol{\xi})
$$

and comparing with (5.2), (5.9) follows by virtue of (5.4). 
The checking of the martingale property for the function pp is very similar to the above and its presentation is therefore omitted.

\section{Appendix}

We present here briefly an argument for proving (2.4).

Let $\mathbf{v}(t)$ be as in Theorem (1.3). Since $\mathbf{x}(t)=\int^{t} \mathbf{v}(s) d s$ is almost surely a $C^{1}$-curve, one may easily convince oneself that we need only show

Clearly

$$
\lim _{\substack{a \rightarrow 0 \\ \phi \rightarrow 0}} v\left(\tau_{\phi \cdot a} \leqq T\right)=0 .
$$

$$
\begin{aligned}
& \bigcap_{\substack{\phi>0 \\
a>0}}\left\{\tau_{\phi, a} \leqq T\right\} \subset T S \equiv\{\text { there exist } s<t \leqq T, \text { such that } \\
& \mathbf{x}(s)=\mathbf{x}(t) \quad \text { and } \quad \mathbf{v}(s)=\mathbf{v}(t) \quad \text { or } \quad \mathbf{v}(s)=-\mathbf{v}(t)\} .
\end{aligned}
$$

Hence we show that tangential selfintersections have probability zero:

Lemma. $v(T S)=0$.

Proof. We shall use the following facts:

(A.1) $(\mathbf{x}(t), \mathbf{v}(t))_{t \geqq 0}$ is Markovian.

(A.2) The conditional probability distribution of the random variable $(\mathbf{x}(t), \mathbf{v}(t))$ given $(\mathbf{x}(s), \mathbf{v}(s))$ is absolutely continuous with bounded density for $t>s$.

(A.3) $v(t)=1$ for all $t$.

(A.4) $\mathbf{v}(t), t \geqq 0$, is Hölder continuous with exponent $\alpha<\frac{1}{2}$.

(A.1) is clear. (A.2) follows from the hypoellipticity of the operator

$$
-\frac{\partial}{\partial t}+\mathbf{v} \cdot \frac{\partial}{\partial \mathbf{x}}+L
$$

(A.3) is clear and (A.4) is a well known fact about Brownian motion.

Let $t_{k} \in[0, T], k \in \mathbb{N}$ be an enumeration of the rationals. Clearly

where

$$
T S=\bigcup_{k} T S(k)
$$

$$
\begin{aligned}
T S(k)= & \left\{\text { there exist } s, t \leqq T, s<t_{k}<t,\right. \text { such that } \\
& \mathbf{x}(s)=\mathbf{x}(t) \text { and } \mathbf{v}(s)=\mathbf{v}(t) \text { or } \mathbf{v}(s)=-\mathbf{v}(t)\},
\end{aligned}
$$

and thus the lemma will follow from

$$
v(T S(k))=0, \quad k \in \mathbb{N} .
$$

By (A.1), $(\mathbf{x}(u), \mathbf{v}(u))_{u<t_{k}}$ and $(\mathbf{x}(u), \mathbf{v}(u))_{u>t_{k}}$ are independent, given $\left(\mathbf{x}\left(t_{k}\right), \mathbf{v}\left(t_{k}\right)\right)$. Hence for the conditional measure

$$
\begin{aligned}
v(T S(k) \mid \mathbf{x}(t)= & \left.\mathbf{q}(t), t \leqq t_{k}\right) \\
\leqq & \lim _{\delta \rightarrow 0} v(\text { there exist } s, t \in[\delta, T] \text { such that } \mathbf{x}(s)=\mathbf{q}(t) \\
& \cdot \text { and } \mathbf{v}(s)=\dot{\mathbf{q}}(t) \text { or } \mathbf{v}(s)=-\dot{\mathbf{q}}(t)) \equiv \lim _{\delta \rightarrow 0} v(T(\mathbf{q}, \delta)),
\end{aligned}
$$


where we used translation invariance of the process and $\mathbf{q}(t), t \leqq T$ is a realisation of $\mathbf{x}(t), t \leqq T$.

We show now that the right-hand side of (A.6) is zero. Then (A.5) follows from integration.

Draw a tube of thickness $2 / n, n \in \mathbb{N}$ around $\mathbf{q}$ and split $[0, T]$ into disjoint intervals $\left[t_{i}^{n}, t_{i+1}^{n}\right)$ of lengths $T / n, i=1, \ldots, n$. At each $t_{i}^{n}$ cut the tube orthogonal to the curve $\mathbf{q}$ to obtain segments $A_{i}^{n}$ and clearly $\left|A_{i}^{n}\right|<C / n^{2}$ for all $i$ and some constant $C$. Now because of (A.3) for $\mathbf{x}$ intersecting $\mathbf{q}$ we must have for some $i$ and $j, \mathbf{x}\left(t_{i}^{n}\right) \in A_{j}^{n}$, and for the intersection to be tangential there must be some $\tilde{t}_{i}^{n} \in I_{i}^{n} \equiv\left[t_{i-1}^{n}, t_{i+1}^{n}\right]$ and some $\tilde{t}_{j}^{n} \in I_{j}^{n}$ for which

$$
\mathbf{v}\left(\tilde{t}_{i}^{n}\right)= \pm \dot{\mathbf{q}}\left(\tilde{t}_{j}^{n}\right)
$$

By (A.4) there is a $K$ such that

$$
\left|\dot{\mathbf{q}}(t)-\dot{\mathbf{q}}\left(\tilde{t}_{j}^{n}\right)\right| \leqq K(2 T / n)^{\alpha}, \quad t \in I_{j}^{n},
$$

and hence

$$
\mathbf{v}\left(\tilde{t}_{i}^{n}\right) \in H_{j}^{n} \equiv\left[-K(2 T / n)^{\alpha} \pm \dot{\mathbf{q}}\left(\tilde{t}_{j}^{n}\right), \pm \dot{\mathbf{q}}\left(\tilde{t}_{j}^{n}\right)+K(2 T / n)^{\alpha}\right] .
$$

Thus we have that

$$
\begin{aligned}
v(T(\mathbf{q}, \delta)) \leqq & \sum_{i, j=1}^{n} v\left(\left\{\mathbf{x}\left(t_{i}^{n}\right) \in A_{j}^{n}\right\} \cap\left\{\mathbf{v}\left(\tilde{t}_{i}^{n}\right) \equiv H_{j}^{n}, \quad \tilde{t}_{i}^{n} \in I_{i}^{n}\right\}\right. \\
& \left.\cap\left\{\sup _{\substack{s, t \leqq T \\
|t-s|<2 T / n}}|\mathbf{v}(t)-\mathbf{v}(s)|<(T / n)^{1 / 4}\right\}\right) \\
& +v\left(\left\{\sup _{\substack{s, t \leqq T \\
|t-s|<2 T / n}}|\mathbf{v}(t)-\mathbf{v}(s)| \geqq(T / n)^{1 / 4}\right\}\right) \\
\leqq & C^{\prime} n^{2} \sup _{j}\left|A_{j}^{n}\right|\left(2\left(K(2 T / n)^{\alpha}+(T / n)^{1 / 4}\right)\right) \\
& +v\left(\left\{\sup _{\substack{s, t \leqq T \\
|t-s|<2 T / n}}|\mathbf{v}(t)-\mathbf{v}(s)| \geqq(T / n)^{1 / 4}\right\}\right)
\end{aligned}
$$

by (A.2). Since $\left|A_{j}^{n}\right| \leqq C / n^{2}$, the first term goes to zero as $n \rightarrow \infty$. For the second term note that for given $K$ and $\alpha>1 / 4$ and $n$ large enough,

$$
\begin{aligned}
& \left\{\sup _{\substack{s, t \leqq T \\
|t-s|<2 T / n}}|\mathbf{v}(t)-\mathbf{v}(s)|<K|t-s|^{\alpha}\right\} \\
& \subset\left\{\sup _{\substack{s, t \leqq T \\
\mid t-s,<2 T / n}}|\mathbf{v}(t)-\mathbf{v}(s)|<K(2 T / n)^{\alpha}\right\} \\
& \subset\left\{\sup _{\substack{s, t \leqq T \\
|t-s|<2 T / n}}|\mathbf{v}(t)-\mathbf{v}(s)|<(T / n)^{1 / 4}\right\} .
\end{aligned}
$$

Now the first set increases with $K$ to the full space, hence the probability of the last set will go to one as $n \rightarrow \infty$, and the probability of its complement will go to zero. 
Acknowledgements. The problem and the earlier version of the proof was discussed with many people. In particular the contributions of B. Israel, S. Kusuoka and H. Spohn are gratefully acknowledged.

\section{References}

1. Kesten, H., Papanicolaou, G.: A limit theorem for stochastic acceleration. Commun. Math. Phys. 78, 19 (1981)

2. Dell'Antonio, G. F.: Large time, small coupling behaviour of a quantum particle in a random field, Ann. Inst. Henri Poincaré XXXIX, 339 (1983)

3. Dürr, D.: $7^{\text {th }}$ International Conference on Mathematical Physics. Boulder/Colorado, Amsterdam: North Holland 1984

4. Stroock, D. W., Varadhan, S. R. S.: Multidimensional diffusion processes. Berlin Heidelberg New York: Springer 1981

5. Calderoni, P., Dürr, D., Kusuoka, S.: in preparation

6. Ikeda, N., Watanabe, S.: Stochastic differential equations and diffusion processes. Amsterdam, New York, Oxford: North Holland 1981

7. Billingsley, P.: Convergence of probability measures. New York: John Wiley 1968

8. DellaCherie, C., Meyer, P. A.: Probabilities and potentials B, Amsterdam: North Holland 1980

9. Dürr, D., Goldstein, S., Lebowitz, J. L.: Z. Wahrscheinlichkeitstheor. Verw. Geb 62, 427 (1983)

10. Estigneev, E.: Theor. Prob. Appl. 22, 563 (1977)

11. Dürr, D., Goldstein, S., Lebowitz, J. L.: Commun. Pure Appl. Math. XXXVIII, 573 (1985)

Communicated by M. Aizenman

Received March 30, 1987 